\title{
On the Piltz divisor problem in algebraic number fields
}

\author{
by \\ ULRICH RAUSCH (Ulm)
}

1. Introduction. Let $K$ be an algebraic number field, and let $k \geq 2$ be a fixed rational integer. Given an ordered system $\left(\mathfrak{K}_{j}\right)=\left(\mathfrak{K}_{1}, \ldots, \mathfrak{K}_{k}\right)$ of $k$ ideal classes (in the widest sense), we consider, for integral ideals $\mathfrak{a} \neq(0)$, the divisor functions

$$
d_{k}\left(\mathfrak{a} ;\left(\mathfrak{K}_{j}\right)\right)=\sum_{\substack{\mathfrak{a}_{1} \ldots \mathfrak{a}_{k}=\mathfrak{a} \\ \mathfrak{a}_{j} \in \mathfrak{K}_{j}(j=1, \ldots, k)}} 1,
$$

the number of representations of $\mathfrak{a}$ as a product of $k$ integral ideals, the $j$ th one lying in the class $\mathfrak{K}_{j}(j=1, \ldots, k)$, and

$$
d_{k}(\mathfrak{a})=\sum_{\mathfrak{a}_{1} \ldots \mathfrak{a}_{k}=\mathfrak{a}} 1,
$$

the number of such representations without any restriction regarding the classes. Clearly,

$$
d_{k}\left(\mathfrak{a} ;\left(\mathfrak{K}_{j}\right)\right)=0 \quad \text { if } \mathfrak{a} \notin \mathfrak{K}_{1} \ldots \mathfrak{K}_{k}
$$

and

$$
d_{k}(\mathfrak{a})=\sum_{\left(\mathfrak{K}_{j}\right)} d_{k}\left(\mathfrak{a} ;\left(\mathfrak{K}_{j}\right)\right),
$$

where the summation is over all systems $\left(\mathfrak{K}_{j}\right)$, of which there are exactly $h^{k}$, $h$ denoting the class number of $K$.

As usual, there are two possible ways of defining summatory functions. The first of these, leading to the Piltz divisor problem for ideals and not dealt with here, is exemplified by

$$
\sum_{N(\mathfrak{a}) \leq x} d_{k}(\mathfrak{a})
$$

taken over those integral ideals $\mathfrak{a} \neq(0)$ in $K$ whose norm $N(\mathfrak{a})$ does not exceed the positive variable $x$. Being basically one-dimensional, sums of this type can be treated with standard tools of analytic number theory such as 
Perron's formula. For results, see [12], [13], [14], and the references given in $[7]$.

The problem we are concerned with in this paper, commonly referred to as the Piltz divisor problem for numbers, is harder in some respects, mainly because of its higher dimensionality and the essential role played by the units of $K$. In detail:

Suppose that $K$ is of degree $[K: \mathbb{Q}]=n=r_{1}+2 r_{2}$ (in the standard notation). Let $d$ denote the discriminant of $K, h$ the class number, $R$ the regulator, $w$ the number of roots of unity, and $r=r_{1}+r_{2}-1$ the number of fundamental units. The conjugates of a number $\nu \in K$ are denoted by $\nu^{(p)}$ $(p=1, \ldots, n)$, and its norm by $N(\nu)$. Let

$$
e_{p}= \begin{cases}1 & \text { for } p=1, \ldots, r_{1} \\ 2 & \text { for } p=r_{1}+1, \ldots, r+1\end{cases}
$$

Further, let $x=\left(x_{1}, \ldots, x_{r+1}\right) \in \mathbb{R}_{+}^{r+1}$ denote a vector of positive real variables and

$$
X=\prod_{p=1}^{r+1} x_{p}^{e_{p}}
$$

We investigate the sums

$$
\begin{aligned}
D_{k}\left(x ;\left(\mathfrak{K}_{j}\right)\right) & =\sum_{0<|\nu(p)| \leq x_{p}} d_{k}\left(\nu ;\left(\mathfrak{K}_{j}\right)\right), \\
D_{k}(x) & =\sum_{0<\left|\nu^{(p)}\right| \leq x_{p}} d_{k}(\nu),
\end{aligned}
$$

extended over all non-zero integers $\nu \in K$ subject to the inequalities $\left|\nu^{(p)}\right| \leq x_{p}(p=1, \ldots, r+1)$. Here we have set

$$
d_{k}\left(\nu ;\left(\mathfrak{K}_{j}\right)\right):=d_{k}\left((\nu) ;\left(\mathfrak{K}_{j}\right)\right), \quad d_{k}(\nu):=d_{k}((\nu)),
$$

$(\nu)$ denoting the principal ideal generated by $\nu$. These functions are known to satisfy asymptotic relations of the form

$$
D_{k}\left(x ;\left(\mathfrak{K}_{j}\right)\right)=A_{k} X P_{k}\left(\log X ;\left(\mathfrak{K}_{j}\right)\right)+\Delta_{k}\left(x ;\left(\mathfrak{K}_{j}\right)\right),
$$

provided $\mathfrak{K}_{1} \ldots \mathfrak{K}_{k}=\mathfrak{K}_{0}$, the principal class, and

$$
D_{k}(x)=h^{k-1} A_{k} X P_{k}(\log X)+\Delta_{k}(x),
$$

where

$$
A_{k}=\frac{1}{(k-1) !}\left(\frac{2^{r_{1}}(2 \pi)^{r_{2}}}{\sqrt{|d|}}\right)^{k}\left(\frac{R}{w}\right)^{k-1},
$$

$P_{k}\left(\cdot ;\left(\mathfrak{K}_{j}\right)\right)$ and $P_{k}(\cdot)$ are certain monic polynomials of degree $k-1$, and the 
$\Delta_{k}$ 's are of order $o(X)$ as $X \rightarrow \infty$. By (1.1) and (1.2),

$$
\Delta_{k}(x)=\sum_{\mathfrak{K}_{1} \ldots \mathfrak{K}_{k}=\mathfrak{K}_{0}} \Delta_{k}\left(x ;\left(\mathfrak{K}_{j}\right)\right) .
$$

The problem now consists in finding good upper and lower estimates for the $\Delta_{k}$ 's.

All results mentioned will be the same for $\Delta_{k}(x)$ and for $\Delta_{k}\left(x ;\left(\mathfrak{K}_{j}\right)\right)$ with $\mathfrak{K}_{1} \ldots \mathfrak{K}_{k}=\mathfrak{K}_{0}$. So, for the sake of brevity, let $\Delta_{k}^{*}(x)$ signify any of these. Grotz [1], in 1980, was the first to give an upper bound for $\left|\Delta_{k}^{*}(x)\right|$, namely

$$
\Delta_{k}^{*}(x) \ll X^{1-1 /\left(\langle k / 2\rangle r_{1}+k r_{2}+1\right)+\delta} \quad(X \geq 1)
$$

for any $\delta>0,\langle k / 2\rangle$ denoting the least rational integer $\geq k / 2$.

The method used runs, in essence, as follows: First, $D_{k}\left(x ;\left(\mathfrak{K}_{j}\right)\right)$ is expressed, by means of Siegel's summation formula, as a series of complex integrals involving a product of Hecke zeta-functions with Grössencharacters. Then the path of integration is shifted into the critical strip, in the course of which the main term occurs as a residue. Finally, the integrals along the new path are estimated, which yields an upper bound for the remainder term. For this purpose, Grotz employed an inequality of Phragmén-Lindelöf type for Hecke's zeta-functions.

Using a more refined summation formula, in 1990 I improved on (1.6) by showing that

$$
\Delta_{k}^{*}(x) \ll X^{1-2 /(n k+2)}(\log X)^{k-n k /(n k+2)} \quad(X \geq 2)
$$

(see [10]). In the same year, Söhne [12], [13] succeeded in giving sharper bounds for Hecke's zeta-functions on the critical line and obtained as a consequence

$$
\Delta_{k}^{*}(x) \ll X^{1-3 /(n k+6)+\delta} \quad(X \geq 1)
$$

for any $\delta>0$, which is better than (1.7) if $n k \geq 7$.

The reader will, however, notice that none of these results, when specialized to the rational field, contains even the elementary estimate $\Delta_{2}(x) \ll$ $x^{1 / 2}$ in the classical Dirichlet divisor problem.

In the present paper we choose a modified approach. We shift the integrals mentioned above further to the left, beyond the critical strip, into the left half-plane. Then, after having transformed the Hecke zeta-functions by means of their functional equation, we apply the summation formula once again, this time "backwards", in order to eliminate the zeta-functions and to recover a series extended over numbers (instead of ideals). The terms of this series are integrals which can be evaluated by the saddle-point method. The result, stated as Lemma 7.1, is an equation for $\Delta_{k}^{*}$ bearing close analogy to 
the Voronol summation formula associated with the classical divisor problem. From this equation, upper and, for the first time, also lower bounds for $\Delta_{k}^{*}$ are derived by arguments similar to those commonly used in the rational case. Our results are:

THEOREM 1.

$$
\Delta_{k}^{*}(x) \ll X^{1-2 /(n k-r+1)}(\log X)^{k-1} \quad(X \geq 2) .
$$

The $\ll$-constant depends only on $K$ and $k$.

Although our method, unlike Söhne's, does, as yet, not involve any nontrivial exponential-sum estimates, this is better than (1.8) if $(k-3) r_{1}+$ $(2 k-3) r_{2} \leq 6$, that is, in the following cases:

- $k=2, r_{2} \leq r_{1}+6$, in particular for all totally real fields;

- $k=3$, for fields with $r_{2} \leq 2$;

- $k=4$, for cubic fields and all totally real fields of degree $\leq 6$;

- $k=5$, for totally real quadratic and cubic fields;

- $k=6$, for real quadratic fields.

For $K=\mathbb{Q}$, Landau's result [4]

$$
\Delta_{k}(x) \ll x^{(k-1) /(k+1)}(\log x)^{k-1}
$$

and in particular Voronoı's $\Delta_{2}(x) \ll x^{1 / 3} \log x$ are recovered.

THEOREM 2.

$$
\Delta_{k}^{*}(x)=\Omega_{ \pm}\left(X^{(n k-r-1) /(2 n k)}\right) \quad \text { as } X \rightarrow \infty .
$$

Theorem 3. If $r_{1}>0$ let $k \not \equiv 1(\bmod 4)$. Then, as $X \rightarrow \infty$,

$$
(-1)^{V} \Delta_{k}^{*}(x)=\Omega_{+}\left((X \log X)^{(n k-r-1) /(2 n k)}(\log \log X)^{k-1}\right),
$$

where $V=r_{1}\left[\frac{k-2}{4}\right]+r_{2}$.

When specialized to the rational field, these theorems give

$$
\Delta_{k}(x)=\Omega_{ \pm}\left(x^{(k-1) /(2 k)}\right),
$$

which is due to Hardy [2], and, for $k \neq \equiv 1(\bmod 4)$,

$$
(-1)^{[(k-2) / 4]} \Delta_{k}(x)=\Omega_{+}\left((x \log x)^{(k-1) /(2 k)}(\log \log x)^{k-1}\right),
$$

which is a special case of Szegö and Walfisz [14]. In particular, Hardy's results

$$
\Delta_{2}(x)=\Omega_{-}\left(x^{1 / 4}\right), \quad \Delta_{2}(x)=\Omega_{+}\left((x \log x)^{1 / 4} \log \log x\right)
$$

on the Dirichlet divisor problem are included.

Except for $k \equiv 1(\bmod 4)$ and apart from sign information, these estimates are not far from the best known today in the rational case, falling short by powers of $\log \log x$ at most. 
One final remark has to be made. The arguments leading to (1.6), (1.7), and (1.8) work equally well when the summation in (1.3) and (1.4) is restricted to totally positive numbers $\nu$, or, what comes to the same thing, when the terms of these sums are multiplied by a sign character. In our method, however, this would cause additional difficulties. I have indicated in the proof of Lemma 7.1 what goes wrong then.

2. A smoothing operator. Given a function $F: \mathbb{R}_{+}^{r+1} \rightarrow \mathbb{C}$, we put for $\varepsilon>0$ and $x \in \mathbb{R}_{+}^{r+1}$,

$$
\mathcal{J}_{\varepsilon} F(x)=(4 \pi \varepsilon)^{-(r+1) / 2} \int_{\mathbb{R}^{r+1}} F\left(x e^{v}\right) e^{-|v|^{2} /(4 \varepsilon)} d v,
$$

where $x e^{v}=\left(x_{1} e^{v_{1}}, \ldots, x_{r+1} e^{v_{r+1}}\right)$ and $|v|=\left(\sum_{p=1}^{r+1} v_{p}^{2}\right)^{1 / 2}$.

Under suitable conditions, the operator $\mathcal{J}_{\varepsilon}$ transforms $F$ into a function $\mathcal{J}_{\varepsilon} F: \mathbb{R}_{+}^{r+1} \rightarrow \mathbb{C}$ which is very well behaved and thus allows straightforward analytical treatment.

In this section we show how to gain information about $F$ from properties of $\mathcal{J}_{\varepsilon} F$. In the first place, we have

Lemma 2.1. Assume that $F: \mathbb{R}_{+}^{r+1} \rightarrow \mathbb{C}$ is locally Lebesgue integrable, that $F$ is continuous at the point $x \in \mathbb{R}_{+}^{r+1}$, and that

$$
\int_{\mathbb{R}^{r+1}}\left|F\left(x e^{v}\right)\right| e^{-|v|^{2} /\left(4 \varepsilon_{0}\right)} d v<\infty
$$

for some $\varepsilon_{0}>0$. Then

$$
\lim _{\varepsilon \rightarrow 0+} \mathcal{J}_{\varepsilon} F(x)=F(x) .
$$

The proof is easy and can be left to the reader.

The next lemma gives a quantitative result. It requires a definition.

The function $\alpha: \mathbb{R}_{+} \rightarrow \mathbb{R}_{+}$is called $\left(\kappa_{1}, \kappa_{2}\right)$-moderately growing $\left(\kappa_{1}>0\right.$, $\left.\kappa_{2} \geq 0\right)$ if

$$
\alpha\left(\xi e^{\xi^{\prime}}\right) \leq \kappa_{1} \alpha(\xi) e^{\kappa_{2}\left|\xi^{\prime}\right|} \quad \text { for all } \xi \in \mathbb{R}_{+} \text {and } \xi^{\prime} \in \mathbb{R} .
$$

Lemma 2.2. Suppose that $\alpha, \beta: \mathbb{R}_{+} \rightarrow \mathbb{R}_{+}$are $\left(\kappa_{1}, \kappa_{2}\right)$-moderately growing and that $F, M: \mathbb{R}_{+}^{r+1} \rightarrow \mathbb{R}$ and $\varepsilon: \mathbb{R}_{+} \rightarrow \mathbb{R}_{+}$satisfy the following conditions for all $x=\left(x_{1}, \ldots, x_{r+1}\right) \in \mathbb{R}_{+}^{r+1}\left(\kappa_{3}, \kappa_{4}, \kappa_{5}\right.$ denote positive real numbers independent of $x)$ :

- $F(x) \geq 0, F(x)$ is non-decreasing with respect to each $x_{p}$;

- $M$ has continuous first-order partial derivatives such that

$$
\left|x_{p} \frac{\partial}{\partial x_{p}} M(x)\right| \leq \kappa_{3} \beta(X) \quad(p=1, \ldots, r+1) ;
$$


- $\varepsilon$ is continuous and $0<\varepsilon(X) \leq 1$;

- $\sqrt{\varepsilon(X)} \cdot \beta(X) \leq \kappa_{4} \alpha(X)$;

- $\left|\mathcal{J}_{\varepsilon(X)}(F-M)(x)\right| \leq \kappa_{5} \alpha(X)$.

Then

$$
F(x)=M(x)+O(\alpha(X)) \quad \text { for all } x \in \mathbb{R}_{+}^{r+1},
$$

where the $O$-constant depends only on $\kappa_{1}, \ldots, \kappa_{5}$ and $n$.

Pr o of. See [10, Theorem 3.1].

In the $\Omega$-direction we have

Lemma 2.3. Let $\alpha: \mathbb{R}_{+} \rightarrow \mathbb{R}_{+}$be $\left(\kappa_{1}, \kappa_{2}\right)$-moderately growing. Let $G: \mathbb{R}_{+}^{r+1} \rightarrow \mathbb{R}$ be a measurable function satisfying

$$
|G(x)| \leq \kappa_{3}\left(X^{\kappa_{4}}+X^{-\kappa_{4}}\right) \quad \text { for } x \in \mathbb{R}_{+}^{r+1}
$$

$\left(\kappa_{3}, \ldots, \kappa_{6}\right.$ denote positive constants $)$. Suppose that for every $X_{0}>1$ there is an $x \in \mathbb{R}_{+}^{r+1}$ and an $\varepsilon>0$ such that

$$
X \geq X_{0}, \quad \varepsilon \leq \kappa_{5}(\log X)^{-1}, \quad \mathcal{J}_{\varepsilon} G(x) \geq \kappa_{6} \alpha(X) .
$$

Then

$$
G(x)=\Omega_{+}(\alpha(X)) \quad \text { as } X \rightarrow \infty .
$$

Proof. $\kappa_{7}, \ldots, \kappa_{14}$ denote positive constants depending only on $\kappa_{1}, \ldots$ $\ldots, \kappa_{6}, \alpha(1)$, and $n$. We assume that, with some $\varrho>0$,

$$
G(x) \leq \varrho \alpha(X) \quad \text { for } X \geq X_{1}>1 .
$$

Let $T>0$ - the exact value of $T$ will be chosen later - and consider $x \in \mathbb{R}_{+}^{r+1}$ with $X \geq e^{n T} X_{1}$. Then, if $|v| \leq T$,

$$
X \exp \left(\sum_{p=1}^{r+1} e_{p} v_{p}\right) \geq X e^{-n|v|} \geq X_{1},
$$

so (2.2) holds also with $x e^{v}$ in place of $x$. Hence

$$
G\left(x e^{v}\right) \leq \varrho \alpha\left(X \exp \left(\sum_{p=1}^{r+1} e_{p} v_{p}\right)\right) \leq \kappa_{1} \varrho \alpha(X) e^{\kappa_{2} n T} \quad \text { for }|v| \leq T .
$$

By (2.1), on the other hand, for all $v \in \mathbb{R}^{r+1}$ we have $\left|G\left(x e^{v}\right)\right| \leq 2 \kappa_{3} X^{\kappa_{4}}$ $\times e^{\kappa_{4} n|v|}$. It follows that

$$
\begin{aligned}
\mathcal{J}_{\varepsilon} G(x) \leq & (4 \pi \varepsilon)^{-(r+1) / 2} \int_{|v| \leq T} \kappa_{1} \varrho \alpha(X) e^{\kappa_{2} n T} e^{-|v|^{2} /(4 \varepsilon)} d v \\
& +(4 \pi \varepsilon)^{-(r+1) / 2} \int_{|v|>T} 2 \kappa_{3} X^{\kappa_{4}} e^{\kappa_{4} n|v|} e^{-T^{2} /(8 \varepsilon)} e^{-|v|^{2} /(8 \varepsilon)} d v \\
\leq & \kappa_{1} \varrho \alpha(X) e^{\kappa_{7} T}+\kappa_{8} X^{\kappa_{4}} e^{-T^{2} /(8 \varepsilon)}
\end{aligned}
$$


if $0<\varepsilon \leq 1$. Now $0<\alpha(1)=\alpha\left(X e^{-\log X}\right) \leq \kappa_{1} \alpha(X) X^{\kappa_{2}}$, hence $1 \leq \kappa_{9} \alpha(X) X^{\kappa_{2}}$. Inserting this yields

$$
\begin{aligned}
\mathcal{J}_{\varepsilon} G(x) & \leq \kappa_{1} \varrho \alpha(X) e^{\kappa_{7} T}+\kappa_{10} \alpha(X) X^{\kappa_{11}} e^{-T^{2} /(8 \varepsilon)} \\
& \leq \kappa_{12} \alpha(X)\left\{\varrho e^{\kappa_{7} T}+X^{\kappa_{11}-T^{2} /\left(8 \kappa_{5}\right)}\right\}
\end{aligned}
$$

if $0<\varepsilon \leq \kappa_{5}(\log X)^{-1}$. The choice $T=\sqrt{8 \kappa_{5}\left(\kappa_{11}+1\right)}$, that is, $\kappa_{11}-$ $T^{2} /\left(8 \kappa_{5}\right)=-1$, then gives

$$
\mathcal{J}_{\varepsilon} G(x) \leq \kappa_{13} \alpha(X)\left\{\varrho+X^{-1}\right\} .
$$

Comparing this to the hypotheses of the lemma, we find that

$$
\kappa_{6} \leq \kappa_{13}\left\{\varrho+X^{-1}\right\}
$$

for arbitrarily large values of $X$. Hence $\varrho \geq \kappa_{14}$, and the assertion follows.

$\mathcal{J}_{\varepsilon}$ can be interchanged with the process of taking residues:

LEMMA 2.4. Let the function $g(s)$ be holomorphic and single-valued for $0<\left|s-s_{0}\right|<\varrho(\varrho>0)$, and let

$$
M(x)=\operatorname{Res}_{s=s_{0}}\left(g(s) X^{s}\right) \quad\left(x \in \mathbb{R}_{+}^{r+1}\right) .
$$

Then

$$
\mathcal{J}_{\varepsilon} M(x)=\operatorname{Res}_{s=s_{0}}\left(g(s) X^{s} \exp \left\{\varepsilon \sum_{p=1}^{r+1} e_{p}^{2} s^{2}\right\}\right) .
$$

Proof. See [10, Lemma 4.1] with $g_{1}=\ldots=g_{r+1}=0$.

3. Summation formulas. Two slightly modified versions of the summation formula from [10] are given. We extend $K$ to a system $\mathfrak{Z}$ of ideal numbers $\widehat{\alpha}, \widehat{\beta}, \ldots$ together with conjugates $\widehat{\alpha}^{(p)}, \widehat{\beta}^{(p)}, \ldots$; for details, see $[3$, Sect. 2]. $\mathfrak{Z}$ splits into a finite number $h$ of classes

$$
\mathfrak{K}=\mathfrak{K}(\widehat{\alpha})=\{\widehat{\alpha} \varrho: \varrho \in K\} \quad(\widehat{\alpha} \neq 0)
$$

which correspond to the ideal classes in the widest sense. Different classes have only the number 0 in common. Regarding notation, we will not distinguish between an ideal class and the corresponding class of ideal numbers.

Assuming $r>0$ for the time being, we consider a free group $\mathcal{U}$ of totally positive units which has finite index $[\mathcal{E}: \mathcal{U}]$ in the full unit group $\mathcal{E}$ of $K$. We fix a basis $\eta_{1}, \ldots, \eta_{r}$ of $\mathcal{U}$ and put

$$
R(\mathcal{U})=\left|\operatorname{det}\left(e_{p} \log \left|\eta_{1}^{(p)}\right|, \ldots, e_{p} \log \left|\eta_{r}^{(p)}\right|\right)_{p=1, \ldots, r}\right| ;
$$

then

$$
[\mathcal{E}: \mathcal{U}]=w R(\mathcal{U}) / R
$$


Given $\tau=\left(\tau_{1}, \ldots, \tau_{r}\right) \in \mathbb{R}^{r}$, we define the generalized Grössencharacter $\lambda_{\tau}$ by

$$
\lambda_{\tau}(\widehat{\nu})=\prod_{p=1}^{r+1}\left|\widehat{\nu}^{(p)}\right|^{i e_{p} E_{p}(\tau)} \quad(0 \neq \widehat{\nu} \in \mathfrak{Z})
$$

with $E_{1}(\tau), \ldots, E_{r+1}(\tau)$ determined by the system of equations

$$
\begin{aligned}
& \sum_{p=1}^{r+1} e_{p} E_{p}(\tau)=0 \\
& \sum_{p=1}^{r+1} e_{p} E_{p}(\tau) \log \left|\eta_{q}^{(p)}\right|=2 \pi \tau_{q} \quad(q=1, \ldots, r) .
\end{aligned}
$$

We further consider a complex-valued arithmetic function $f$ defined on the non-zero ideal integers $\widehat{\nu} \in \mathfrak{Z}$ (e.g., $f(\widehat{\nu})=0$ outside a particular class $\mathfrak{K})$, such that always

$$
f(\eta \widehat{\nu})=f(\widehat{\nu}) \quad(\eta \in \mathcal{U}) .
$$

Clearly, this invariance property is shared by $\lambda_{\tau}$ if and only if $\tau=m \in \mathbb{Z}^{r}$. In that case, the Dirichlet series

$$
\Xi\left(s ; \lambda_{m} f, \mathcal{U}\right)=\sum_{(\hat{\nu})_{\mathcal{U}}}^{\prime} \frac{\lambda_{m}(\widehat{\nu}) f(\widehat{\nu})}{|N(\widehat{\nu})|^{s}} \quad(s \in \mathbb{C})
$$

is well-defined; here the summation is over a complete set of ideal integers $\widehat{\nu} \neq 0$ which are not associated with respect to $\mathcal{U}$.

We shall frequently use abbreviations like

$$
|\widehat{\nu}| x=\left(\left|\widehat{\nu}^{(1)}\right| x_{1}, \ldots,\left|\widehat{\nu}^{(r+1)}\right| x_{r+1}\right) .
$$

LEMma 3.1. Let the measurable function $\Phi: \mathbb{R}_{+}^{r+1} \rightarrow \mathbb{C}$ and the number $\sigma \in \mathbb{R}$ be such that

$$
\int_{\mathbb{R}_{+}^{r+1}}|\Phi(u)| \prod_{p=1}^{r+1} u_{p}^{e_{p} \sigma-1} d u<\infty \quad \text { and } \quad \sum_{(\hat{\nu})_{\mathcal{U}}}^{\prime} \frac{|f(\widehat{\nu})|}{|N(\widehat{\nu})|^{\sigma}}<\infty .
$$

Then the series

$$
F(x)=\sum_{\hat{\nu}}^{\prime} \lambda_{\tau}(\widehat{\nu}) f(\widehat{\nu}) \Phi(|\widehat{\nu}| x),
$$

extended over all integers $\widehat{\nu} \neq 0$ in $\mathfrak{Z}$, is absolutely convergent for almost all $x \in \mathbb{R}_{+}^{r+1}$. For all $x \in \mathbb{R}_{+}^{r+1}$ and all $\varepsilon>0, \mathcal{J}_{\varepsilon} F(x)$ is absolutely convergent 
and

$$
\begin{aligned}
\mathcal{J}_{\varepsilon} F(x)= & \frac{1}{2 \pi i R(\mathcal{U})} \sum_{m} \int_{(\sigma)} \Psi\left(s-i E_{1}(m-\tau), \ldots, s-i E_{r+1}(m-\tau)\right) \\
& \times \Xi\left(s ; \lambda_{m} f, \mathcal{U}\right) \prod_{p=1}^{r+1} x_{p}^{-e_{p}\left(s-i E_{p}(m-\tau)\right)} \\
& \times \exp \left\{\varepsilon \sum_{p=1}^{r+1} e_{p}^{2}\left(s-i E_{p}(m-\tau)\right)^{2}\right\} d s
\end{aligned}
$$

where series and integrals on the right also converge absolutely. Here $m$ ranges over $\mathbb{Z}^{r}$, the integration is along the vertical line $\operatorname{Re} s=\sigma$, and $\Psi$ is the Mellin transform of $\Phi$ :

$$
\Psi\left(s_{1}, \ldots, s_{r+1}\right)=2^{r_{2}} \int_{\mathbb{R}_{+}^{r+1}} \Phi(u) \prod_{p=1}^{r+1} u_{p}^{e_{p} s_{p}-1} d u .
$$

Proof. Apart from the trivial extension to ideal numbers, this is a special case of [10, Theorem 2.2].

The second version deals with the case of a given function $\Psi$ which is not necessarily representable as a Mellin transform.

LEMMA 3.2. Let $\sigma \in \mathbb{R}$ be such that

$$
\sum_{(\hat{\nu})_{\mathcal{U}}}^{\prime} \frac{|f(\widehat{\nu})|}{|N(\widehat{\nu})|^{\sigma}}<\infty .
$$

Let the complex-valued function $\Psi\left(s_{1}, \ldots, s_{r+1}\right)$ be defined and continuous on the $(r+1)$-space given by $s_{p}=\sigma+i t_{p}, t_{p} \in \mathbb{R}(p=1, \ldots, r+1)$, and suppose that

$$
\left|\Psi\left(s_{1}, \ldots, s_{r+1}\right)\right| \leq \kappa_{1} \exp \left(\kappa_{2} \sum_{p=1}^{r+1}\left|t_{p}\right|\right)
$$

with $\kappa_{1}, \kappa_{2}>0$ independent of $t_{1}, \ldots, t_{r+1}$. Then, for $u, x \in \mathbb{R}_{+}^{r+1}$ and $\varepsilon>0$, the integral

$$
\begin{aligned}
\widetilde{\Phi}_{\varepsilon}(u)= & (2 \pi i)^{-(r+1)} \int_{(\sigma)} \ldots \int_{(\sigma)} \Psi\left(s_{1}, \ldots, s_{r+1}\right) \\
& \times \prod_{p=1}^{r+1} u_{p}^{-e_{p} s_{p}} \cdot \exp \left\{\varepsilon \sum_{p=1}^{r+1} e_{p}^{2} s_{p}^{2}\right\} d s_{1} \ldots d s_{r+1}
\end{aligned}
$$


and the series

$$
\begin{aligned}
G(x, \varepsilon, \tau)= & \frac{1}{2 \pi i R(\mathcal{U})} \sum_{m} \int_{(\sigma)} \Psi\left(s-i E_{1}(m-\tau), \ldots, s-i E_{r+1}(m-\tau)\right) \\
& \times \Xi\left(s ; \lambda_{m} f, \mathcal{U}\right) \prod_{p=1}^{r+1} x_{p}^{-e_{p}\left(s-i E_{p}(m-\tau)\right)} \\
& \times \exp \left\{\varepsilon \sum_{p=1}^{r+1} e_{p}^{2}\left(s-i E_{p}(m-\tau)\right)^{2}\right\} d s
\end{aligned}
$$

are absolutely convergent. For those $x$ and $\varepsilon$ for which the series

$$
F(x, \varepsilon, \tau)=\sum_{\hat{\nu}}^{\prime} \lambda_{\tau}(\widehat{\nu}) f(\widehat{\nu}) \widetilde{\Phi}_{\varepsilon}(|\widehat{\nu}| x)
$$

is also absolutely convergent, we have the equality $F(x, \varepsilon, \tau)=G(x, \varepsilon, \tau)$.

Proof. We first prove that

$$
\begin{aligned}
G_{0}(x, \varepsilon, \tau)= & \frac{1}{2 \pi i R(\mathcal{U})} \sum_{m} \int_{(\sigma)} \Psi\left(s-i E_{1}(m-\tau), \ldots, s-i E_{r+1}(m-\tau)\right) \\
& \times \prod_{p=1}^{r+1} x_{p}^{-e_{p}\left(s-i E_{p}(m-\tau)\right)} \cdot \exp \left\{\varepsilon \sum_{p=1}^{r+1} e_{p}^{2}\left(s-i E_{p}(m-\tau)\right)^{2}\right\} d s
\end{aligned}
$$

equals

$$
F_{0}(x, \varepsilon, \tau)=\sum_{\eta \in \mathcal{U}} \lambda_{\tau}(\eta) \widetilde{\Phi}_{\varepsilon}(|\eta| x)
$$

whenever the last series converges absolutely. To this end we keep $x$ and $\varepsilon$ fixed and regard $\tau$ as variable. Using the estimate [10, (1.6)]

$$
\sum_{p=1}^{r+1} e_{p}^{2}\left(t-E_{p}(\tau)\right)^{2} \geq \kappa_{3}\left(t^{2}+\sum_{q=1}^{r} \tau_{q}^{2}\right),
$$

we find that $G_{0}$ is absolutely convergent, and that the convergence is uniform with respect to $\tau$ in any bounded set. Hence $G_{0}$ represents a continuous function of $\tau$ which clearly is periodic with period 1 in each $\tau_{q}$.

On the other hand, the same calculations as in the proof of $[10$, Theorem 2.1] show that $F_{0}$ is in fact the Fourier expansion of $G_{0}$ with respect to $\tau$; note that

$$
\lambda_{\tau}(\eta)=\exp \left(2 \pi i\left(l_{1} \tau_{1}+\ldots+l_{r} \tau_{r}\right)\right) \quad \text { if } \eta=\eta_{1}^{l_{1}} \ldots \eta_{r}^{l_{r}}, l_{q} \in \mathbb{Z} .
$$

Thus absolute convergence of $F_{0}$ implies $F_{0}=G_{0}$. 
Now, if the series $F$ is absolutely convergent, it can be rearranged to

$$
F(x, \varepsilon, \tau)=\sum_{(\hat{\nu}) \mathcal{U}_{\mathcal{U}}}^{\prime} \lambda_{\tau}(\widehat{\nu}) f(\widehat{\nu}) F_{0}(|\widehat{\nu}| x, \varepsilon, \tau)
$$

with all the inner $F_{0}$ 's converging absolutely as well. To complete the proof, we replace the $F_{0}$ 's by the corresponding $G_{0}$ 's and move the summation with respect to $(\widehat{\nu})_{\mathcal{U}}$ under the integral sign, as we may by absolute convergence.

The above results also hold for $r=0$ if we then put $\mathcal{U}=\{1\}, R(\mathcal{U})=1$, $\tau=0, E_{1}(0)=0$, and keep in the sum over $m$ only the term with $m=0$; cf. [10, Sect. 2].

In this sense, the following computations will always include the case $r=0$.

4. The generating Dirichlet series. Let $\mathcal{U}$ be any unit group as specified in Section 3, and let $\lambda_{m}, m \in \mathbb{Z}^{r}$, be a corresponding Grössencharacter according to (3.2). Then, given a system $\left(\mathfrak{K}_{j}\right)=\left(\mathfrak{K}_{1}, \ldots, \mathfrak{K}_{k}\right)$ of ideal classes, we consider the analytic function defined for $\operatorname{Re} s>1$ by

$$
Z_{k}\left(s ; \lambda_{m},\left(\mathfrak{K}_{j}\right)\right)=\frac{1}{R(\mathcal{U})} \sum_{(\hat{\nu})_{\mathcal{U}}}^{\prime} \frac{\lambda_{m}(\widehat{\nu}) d_{k}\left(\widehat{\nu} ;\left(\mathfrak{K}_{j}\right)\right)}{|N(\widehat{\nu})|^{s}},
$$

the summation being over a complete set of non-zero integers $\widehat{\nu} \in \mathfrak{Z}$ which are not associated with respect to $\mathcal{U}$. Remember that $d_{k}\left(\widehat{\nu} ;\left(\mathfrak{K}_{j}\right)\right):=$ $d_{k}\left((\widehat{\nu}) ;\left(\mathfrak{K}_{j}\right)\right)=0$ unless $\widehat{\nu} \in \mathfrak{K}_{1} \ldots \mathfrak{K}_{k}$.

LEMMA 4.1. $Z_{k}\left(s ; \lambda_{m},\left(\mathfrak{K}_{j}\right)\right)$ is an entire function, except for $m=0$ when its only singularity in $\mathbb{C}$ is a $k$-fold pole at $s=1$ such that

$$
\lim _{s \rightarrow 1}(s-1)^{k} Z_{k}\left(s ; 1,\left(\mathfrak{K}_{j}\right)\right)=\left(\frac{2^{r_{1}}(2 \pi)^{r_{2}}}{\sqrt{|d|}}\right)^{k}\left(\frac{R}{w}\right)^{k-1} .
$$

We have the functional equation

$$
\begin{aligned}
Z_{k}\left(s ; \lambda_{m},\left(\mathfrak{K}_{j}\right)\right) & \left(\frac{|d|}{4^{r_{2}} \pi^{n}}\right)^{k / 2} \prod_{p=1}^{r+1}\left(\frac{e_{p} \pi}{\left|\widehat{\delta}^{(p)}\right|}\right)^{e_{p} k\left(s-i E_{p}(m)\right)} \\
& \quad \times \prod_{p=1}^{r+1}\left(\frac{\Gamma\left(\frac{e_{p}}{2}\left(1-s+i E_{p}(m)\right)\right)}{\Gamma\left(\frac{e_{p}}{2}\left(s-i E_{p}(m)\right)\right)}\right)^{k} \cdot Z_{k}\left(1-s ; \lambda_{-m},\left(\mathfrak{K}_{j}^{\prime}\right)\right),
\end{aligned}
$$

in which $(\widehat{\delta})=\mathfrak{d}$ is the different of $K$, and the class $\mathfrak{K}_{j}^{\prime}$ is determined by $\mathfrak{K}_{j} \cdot \mathfrak{K}_{j}^{\prime}=\mathfrak{K}(\widehat{\delta})(j=1, \ldots, k)$. 
Proof. We assume first that $\lambda_{m}(\eta)=1$ for every unit $\eta \in \mathcal{E}$. Then, collecting associated numbers and using (3.1), we find that

$$
Z_{k}\left(s ; \lambda_{m},\left(\mathfrak{K}_{j}\right)\right)=\frac{w}{R} \prod_{j=1}^{k} \zeta\left(s ; \lambda_{m}, \mathfrak{K}_{j}\right),
$$

where $\zeta\left(s ; \lambda_{m}, \mathfrak{K}_{j}\right)$ is Hecke's zeta function:

$$
\zeta\left(s ; \lambda_{m}, \mathfrak{K}\right)=\sum_{(\hat{\mu}) \in \mathfrak{K}}^{\prime} \frac{\lambda_{m}(\widehat{\mu})}{|N(\widehat{\mu})|^{s}} \quad(\operatorname{Re} s>1),
$$

summed over all integral ideals $(\widehat{\mu}) \neq(0)$ in the class $\mathfrak{K}$. According to Hecke [3] (see also Landau [6, Sect. 13]), $\zeta\left(s ; \lambda_{m}, \mathfrak{K}\right)$ is holomorphic throughout $\mathbb{C}$, except that for $m=0$ it has a simple pole at $s=1$ with residue

$$
\frac{2^{r_{1}}(2 \pi)^{r_{2}} R}{\sqrt{|d|} w}
$$

There is a functional equation which can be put in the form

$$
\xi\left(s ; \lambda_{m}, \mathfrak{K}\right)=\lambda_{m}(\widehat{\delta}) \xi\left(1-s ; \lambda_{-m}, \mathfrak{K}^{\prime}\right),
$$

where

$$
\begin{gathered}
\xi\left(s ; \lambda_{m}, \mathfrak{K}\right)=\gamma\left(\lambda_{m}\right) \Gamma\left(s ; \lambda_{m}\right) A^{s} \zeta\left(s ; \lambda_{m}, \mathfrak{K}\right), \\
A=2^{-r_{2}} \pi^{-n / 2}|d|^{1 / 2}, \quad \gamma\left(\lambda_{m}\right)=\prod_{p=1}^{r+1} e_{p}^{i e_{p} E_{p}(m) / 2}, \\
\Gamma\left(s ; \lambda_{m}\right)=\prod_{p=1}^{r+1} \Gamma\left(\frac{e_{p}}{2}\left(s-i E_{p}(m)\right)\right), \quad \text { and } \quad \mathfrak{K} \cdot \mathfrak{K}^{\prime}=\mathfrak{K}(\widehat{\delta}) .
\end{gathered}
$$

Now (4.1) follows after a simple calculation using $|N(\widehat{\delta})|=|d|$. On the other hand, the assertion holds trivially if $\lambda_{m}(\eta) \neq 1$ for some $\eta \in \mathcal{E}$, since then $Z_{k}\left(s ; \lambda_{m},\left(\mathfrak{K}_{j}\right)\right)=0$ identically.

Estimating $\zeta\left(s ; \lambda_{m}, \mathfrak{K}\right)$ by means of the Phragmén-Lindelöf principle, as demonstrated by Rademacher $[8$, Sect. 8$]$ in a very similar case, and inserting in (4.2), we get

Lemma 4.2. Let $0<\varrho \leq 1 / 2, \sigma=\operatorname{Re} s, t=\operatorname{Im} s$. Then

$$
Z_{k}\left(s ; \lambda_{m},\left(\mathfrak{K}_{j}\right)\right) \ll \prod_{p=1}^{r+1}\left(1+\left|t-E_{p}(m)\right|\right)^{e_{p} k(1+\varrho-\sigma) / 2}
$$

if $-\varrho \leq \sigma \leq 1+\varrho$ and $|s-1| \geq 1 / 4$, the $\ll$-constant depending only on $K$, $k$, and $\varrho$. 
5. Asymptotic evaluation of integrals. We investigate the behaviour of

$$
I_{\varepsilon}(B ; \psi)=\frac{1}{2 \pi i} \int_{(\sigma)} \psi(s) B^{-s} e^{\varepsilon s^{2}} d s \quad(\sigma>0)
$$

for $B>0$ and $\varepsilon>0$, when the analytic function $\psi$ is subject to certain conditions (which guarantee in particular that the value of the integral is independent of $\sigma>0)$.

This involves a fairly lengthy application of the saddle-point method. Fortunately we can, after a suitable change of variables, take most of the computations without alteration from [9], where a special case is studied.

LEMma 5.1. Let $\psi(s)$ be holomorphic on

$$
D=\{s \in \mathbb{C}: s \neq 0,|\arg s| \leq 3 \pi / 4\}
$$

and suppose that

$$
\psi(\bar{s})=\overline{\psi(s)}
$$

for $s \in D$. Suppose further that there are real constants $c, \alpha, \beta, \gamma, \delta$ with $\alpha>0, \gamma>0, \beta \geq 0$ such that

$$
\begin{aligned}
& \psi(s) \\
= & c e^{i \delta} \exp (\alpha s(\log (s / \gamma)-1-\pi i / 2)+(\beta-1) \log s) \cdot\left\{1+O\left(\frac{1}{t+1}\right)\right\}
\end{aligned}
$$

for $s \in D$ with $t=\operatorname{Im} s \geq 0$ and sufficiently large $|s|$. Then

$$
\begin{aligned}
I_{\varepsilon}(B ; \psi)= & c \sqrt{\frac{2}{\pi \alpha}} e^{-\varepsilon b^{2}} b^{\beta-1 / 2} \cos \left(\alpha b-\frac{\pi}{2} \beta+\frac{\pi}{4}-\delta\right) \\
& +O\left(e^{-\varepsilon b^{2} / 2} b^{\beta-1}\right)+O\left(b^{\beta} e^{-\log ^{2}(\alpha b / 2)}\right)
\end{aligned}
$$

for $b:=\gamma B^{1 / \alpha} \geq 2 / \alpha$ and $0<\varepsilon \leq \alpha^{2} / 8$. The $O$-constants are independent of $B$ and $\varepsilon$.

Proof. We may assume that (5.3) holds uniformly in the region $s \in D$, $t \geq 0,|s| \geq(2 \alpha)^{-1}$. The substitution $s \rightarrow b s$ yields

$$
I_{\varepsilon}(B ; \psi)=b^{\beta} \cdot \frac{1}{2 \pi i} \int_{(\sigma)} H(s) d s \quad(\sigma>0),
$$

where

$$
H(s)=b^{-(\beta-1)} \psi(b s) B^{-b s} e^{\varepsilon b^{2} s^{2}} .
$$


By (5.3) we have

$$
\begin{aligned}
H(s)= & c e^{i \delta} \exp \left(\alpha b s(\log s-1-\pi i / 2)+(\beta-1) \log s+\varepsilon b^{2} s^{2}\right) \\
& \times\left\{1+O\left(\frac{1}{b t+1}\right)\right\} \\
= & c e^{i \delta} \exp \left(2 b_{0} s(\log s-1-\pi i / 2)+(\beta-1) \log s+\varepsilon_{0} b_{0}^{2} s^{2}\right) \\
& \times\left\{1+O\left(\frac{1}{b_{0} t+1}\right)\right\}
\end{aligned}
$$

if $b_{0}=\alpha b / 2$ and $\varepsilon_{0}=(2 / \alpha)^{2} \varepsilon$, valid in the region $s \in D, t \geq 0,|s| \geq$ $(2 \alpha b)^{-1}=\left(4 b_{0}\right)^{-1}$. But, apart from the constant factor $c e^{i \delta}$, this is formula (9) of [9]. Evidently, the calculations leading to the proof of [9, Hilfssatz 3] apply to any function $H(s)$ satisfying this asymptotic relation.

Hence, if we define the contours $W_{1}, W_{2}, W_{3}$, and $W$ as in [9] and use (5.2), we get

$$
I_{\varepsilon}(B ; \psi)=b^{\beta} \cdot \frac{1}{2 \pi i} \int_{W} H(s) d s=\frac{1}{\pi} b^{\beta} \cdot \operatorname{Im}\left(\int_{W_{1}}+\int_{W_{2}}+\int_{W_{3}}\right) H(s) d s
$$

and

$$
\begin{aligned}
\left(\int_{W_{1}}+\int_{W_{2}}+\int_{W_{3}}\right) H(s) d s= & c e^{i \delta} \sqrt{\pi} e^{-\varepsilon_{0} b_{0}^{2}} b_{0}^{-1 / 2} e^{-i\left(2 b_{0}-\pi \beta / 2-\pi / 4\right)} \\
& +O\left(e^{-\varepsilon_{0} b_{0}^{2} / 2} b_{0}^{-1}\right)+O\left(e^{-\log ^{2} b_{0}}\right),
\end{aligned}
$$

provided $b_{0} \geq 1$ and $0<\varepsilon_{0} \leq 1 / 2$. The assertion follows.

For small values of $B$ we have

LEMMA 5.2. In addition to the assumptions of Lemma 5.1, suppose that $\psi$ is holomorphic and single-valued on

$$
D_{0}=D \cup\left\{s \in \mathbb{C}: \operatorname{Re} s \geq-\varrho_{0}\right\} \quad\left(\varrho_{0}>0\right)
$$

with the possible exception of singularities at the points $s_{1}, \ldots, s_{l}$ lying in the real interval $-\varrho_{0}<s_{j} \leq 0(j=1, \ldots, l)$. Then, if $B_{1}>0$ and $\varepsilon_{1}>0$ are arbitrary but fixed,

$$
I_{\varepsilon}(B ; \psi)=\sum_{j=1}^{l} \operatorname{Res}_{s=s_{j}}\left(\psi(s) B^{-s} e^{\varepsilon s^{2}}\right)+O\left(B^{\varrho_{0}}\right)
$$

uniformly for $0<B \leq B_{1}$ and $0<\varepsilon \leq \varepsilon_{1}$.

P r o o f. We proceed as in the proof of [9, Hilfssatz 4] (incidentally putting right some mixed-up plus and minus signs on page 391, line 2, of [9]).

We replace in (5.1) the line of integration by the boundary of $D_{0}$, taking account of the residues at $s_{1}, \ldots, s_{l}$. The contribution of the straight line 
from $(-1-i) \varrho_{0}$ to $(-1+i) \varrho_{0}$ is clearly $\ll B^{\varrho_{0}}$. On the line

$$
\mathcal{C}: s=(-1+i) \varrho=\sqrt{2} e^{3 \pi i / 4} \varrho \quad\left(\varrho \geq \varrho_{0}\right)
$$

we have $\operatorname{Re} s^{2}=0$ and

$$
\operatorname{Re}(\alpha s(\log (s / \gamma)-1-\pi i / 2))=-\alpha \varrho(\log \varrho+\log (\sqrt{2} / \gamma)-1+\pi / 4) .
$$

Hence, by (5.3) (which we may assume to hold throughout $\mathcal{C}$ ),

$$
\int_{\mathcal{C}} \psi(s) B^{-s} e^{\varepsilon s^{2}} d s \ll \int_{\varrho_{0}}^{\infty} e^{-\alpha \varrho \log \varrho+\kappa \varrho+(\beta-1) \log \varrho}\left(B / B_{1}\right)^{\varrho} d \varrho,
$$

where $\kappa>0$ is independent of $\varrho, B$, and $\varepsilon$. If $B / B_{1} \leq 1$, the last integral is $\ll B^{\varrho_{0}}$. By (5.2), an analogous result holds in the lower half-plane. So the lemma is proved.

The special case we are interested in at present is dealt with in

LEMMA 5.3. Let

$$
\psi(s)=\frac{1}{s}\left(\frac{\Gamma((s+a) / 2)}{\Gamma(-s / 2)}\right)^{k},
$$

where $a \geq 0$. Then, for $B>0,0<\varepsilon \leq k^{2} / 8$, and any $\kappa>0$,

$$
\begin{aligned}
I_{\varepsilon}(B ; \psi)= & \frac{1}{\sqrt{k \pi}} e^{-4 \varepsilon B^{2 / k}} B^{(a k-1) /(2 k)} \cos \left(2 k B^{1 / k}+(2-a) \frac{k \pi}{4}+\frac{\pi}{4}\right) \\
& +O\left(e^{-2 \varepsilon B^{2 / k}} B^{(a k-2) /(2 k)}\right)+O\left((B+1)^{-\kappa}\right) .
\end{aligned}
$$

The $O$-constants depend only on $k, a$, and $\kappa$.

$\operatorname{Proof}$. Let $|s| \geq 1,|\arg s| \leq 3 \pi / 4$, and $t=\operatorname{Im} s \geq 0$. Then

$$
\log \Gamma((s+a) / 2)=\frac{1}{2}(s+a-1) \log \frac{s}{2}-\frac{s}{2}+\log \sqrt{2 \pi}+O\left(\frac{1}{|s|}\right)
$$

by Stirling's formula (cf. also [9, p. 385 et seq.]), and

$$
\sin \frac{\pi s}{2}=\frac{i}{2} e^{-\pi i s / 2}\left\{1+O\left(\frac{1}{t+1}\right)\right\} .
$$

It follows that

$$
\begin{aligned}
\psi(s)= & \frac{1}{s}\left(-\frac{1}{\pi} \Gamma\left(\frac{s+a}{2}\right) \Gamma\left(\frac{s+2}{2}\right) \sin \frac{\pi s}{2}\right)^{k} \\
= & 2^{-a k / 2} e^{-k \pi i / 2} \exp \left(k s\left(\log \frac{s}{2}-1-\frac{\pi i}{2}\right)+\left(\frac{a k}{2}-1\right) \log s\right) \\
& \times\left\{1+O\left(\frac{1}{t+1}\right)\right\} .
\end{aligned}
$$


Thus, application of Lemma 5.1 with $c=2^{-a k / 2}, \alpha=k, \beta=a k / 2$, $\gamma=2, \delta=-k \pi / 2$, and $b=2 B^{1 / k}$ proves the assertion when $B \geq k^{-k}$, since the second $O$-term in (5.4) is clearly $\ll(B+1)^{-\kappa}$.

On the other hand, $\psi(s)$ is holomorphic for $\operatorname{Re} s>-a$ if $a>0$, and is holomorphic for $\operatorname{Re} s>-2$ except for a single pole at $s=0$ if $a=0$. So, in both cases, Lemma 5.2 shows that $I_{\varepsilon}(B ; \psi)$ is bounded for $0<B \leq k^{-k}$. In that range, the assertion therefore holds trivially.

6. Series estimations. As before, let $\left(\mathfrak{K}_{j}\right)=\left(\mathfrak{K}_{1}, \ldots, \mathfrak{K}_{k}\right)$ be a system of ideal classes.

LEMma 6.1. Let $\{1,2, \ldots, r+1\}=T_{1} \cup T_{2}, T_{1} \cap T_{2}=\emptyset$, and let $0 \leq y_{p} \leq 1$ for $p \in T_{1}$. With the functions $\Phi_{p}: \mathbb{R}_{+} \rightarrow \mathbb{R}_{+}$given by

$$
\Phi_{p}(u)= \begin{cases}e^{-u^{2 / k}} u^{-e_{p} y_{p}} & \text { if } p \in T_{1}, \\ (u+1)^{-e_{p}} u^{-e_{p}} & \text { if } p \in T_{2},\end{cases}
$$

let

$$
S(x)=\sum_{\hat{\nu}}^{\prime} d_{k}\left(\widehat{\nu} ;\left(\mathfrak{K}_{j}\right)\right) \prod_{p=1}^{r+1} \Phi_{p}\left(\left|\widehat{\nu}^{(p)}\right| x_{p}\right)
$$

for $x \in \mathbb{R}_{+}^{r+1}$, the sum being taken over all integers $\widehat{\nu} \neq 0$ in $\mathfrak{Z}$ (or, equivalently, in $\left.\mathfrak{K}_{1} \ldots \mathfrak{K}_{k}\right)$. Then, for any $\delta$ with $0<\delta<1$,

$$
S(x) \ll X^{-1-\delta},
$$

where the $\ll$-constant depends only on $K, k$, and $\delta$.

Pr o of. We apply Lemma 3.1. The Mellin transform in question is

$$
\Psi\left(s_{1}, \ldots, s_{r+1}\right)=2^{r_{2}} \prod_{p=1}^{r+1} \Psi_{p}\left(s_{p}\right),
$$

where

$$
\begin{aligned}
\Psi_{p}(s) & =\int_{0}^{\infty} e^{-u^{2 / k}} u^{e_{p}\left(s-y_{p}\right)-1} d u \\
& =\frac{k}{2} \Gamma\left(\frac{e_{p} k}{2}\left(s-y_{p}\right)\right) \quad \text { if } p \in T_{1}, \operatorname{Re} s>y_{p}
\end{aligned}
$$

and

$$
\begin{aligned}
\Psi_{p}(s) & =\int_{0}^{\infty}(u+1)^{-e_{p}} u^{e_{p}(s-1)-1} d u \\
& =\Gamma\left(e_{p}(s-1)\right) \Gamma\left(e_{p}(2-s)\right) \quad \text { if } p \in T_{2}, 1<\operatorname{Re} s<2 .
\end{aligned}
$$


Hence Lemma 3.1 tells us that

$$
\text { (6.1) } \begin{aligned}
\mathcal{J}_{\varepsilon} S(x)= & \frac{2^{r_{2}}}{2 \pi i} \sum_{m} \int_{(\sigma)} \prod_{p=1}^{r+1} \Psi_{p}\left(s-i E_{p}(m)\right) \cdot Z_{k}\left(s ; \lambda_{m},\left(\mathfrak{K}_{j}\right)\right) \\
& \times \prod_{p=1}^{r+1} x_{p}^{-e_{p}\left(s-i E_{p}(m)\right)} \cdot \exp \left\{\varepsilon \sum_{p=1}^{r+1} e_{p}^{2}\left(s-i E_{p}(m)\right)^{2}\right\} d s
\end{aligned}
$$

for $1<\sigma<2$ and $\varepsilon>0$, and that $S(x)$ converges for almost all $x \in \mathbb{R}_{+}^{r+1}$. But, since the $\Phi_{p}$ 's are decreasing, this implies already that $S(x)$ is uniformly convergent on every compact subset of $\mathbb{R}_{+}^{r+1}$. Hence $S: \mathbb{R}_{+}^{r+1} \rightarrow \mathbb{R}_{+}$is continuous, and thus $\lim _{\varepsilon \rightarrow 0+} \mathcal{J}_{\varepsilon} S(x)=S(x)$ by Lemma 2.1 .

On the other hand, as a consequence of

$$
\Gamma(s) \ll e^{-\pi|t| / 2}|t|^{\sigma-1 / 2} \ll e^{-|t|} \quad(s=\sigma+i t)
$$

(see for example [6, Satz 160]), we have

$$
\Psi_{p}(s) \ll e^{-e_{p}|t|} \quad(p=1, \ldots, r+1)
$$

uniformly in any region $\sigma_{1} \leq \sigma \leq \sigma_{2},|t| \geq 1$. Further (cf. [10, p. 50]),

$$
\sum_{p=1}^{r+1} e_{p}\left|t-E_{p}(m)\right| \geq \kappa\left(|t|+\sum_{q=1}^{r}\left|m_{q}\right|\right),
$$

where $\kappa>0$ depends only on the basis $\eta_{1}, \ldots, \eta_{r}$ of $\mathcal{U}$ underlying the definition of the numbers $E_{p}(m)$. It follows that the right-hand side of (6.1) is absolutely convergent also for $\varepsilon=0$; hence

$$
\begin{aligned}
S(x)=\frac{2^{r_{2}}}{2 \pi i} \sum_{m} \int_{(\sigma)} \prod_{p=1}^{r+1} \Psi_{p}\left(s-i E_{p}(m)\right) & Z_{k}\left(s ; \lambda_{m},\left(\mathfrak{K}_{j}\right)\right) \\
& \times \prod_{p=1}^{r+1} x_{p}^{-e_{p}\left(s-i E_{p}(m)\right)} d s
\end{aligned}
$$

for $1<\sigma<2$, and the choice $\sigma=1+\delta$ yields the assertion.

LEMMA 6.2. In the notation of Lemma 6.1 , suppose that $T_{2}=\emptyset$ and $0 \leq y_{p} \leq 3 / 4$ for $p=1, \ldots, r+1$. Then

$$
S(x) \ll X^{-1}(|\log X|+1)^{k-1} \quad \text { for all } x \in \mathbb{R}_{+}^{r+1}
$$

and

$$
S(x) \gg X^{-1}|\log X|^{k-1} \quad \text { if } X \leq c_{1},
$$

where the positive constant $c_{1} \leq 1 / 2$, as well as the constants implied by the symbols $\ll$ and $\gg$, depends only on $K$ and $k$.

Pr o of. The case $X \geq 1$ being covered by Lemma 6.1 , we assume $X<1$. In view of $(6.2),(6.3)$, and Lemma 4.2 , we may move in (6.4) the path of 
integration to the line $\operatorname{Re} s=4 / 5$, if we take account of the $k$-fold pole at $s=1$ occurring for $m=0$. The sum of the integrals along the new path is $\ll X^{-4 / 5}$, and the residue at $s=1$ has the form $X^{-1} Q(-\log X)$, where $Q$ is a polynomial of degree $k-1$ which depends on $K, k,\left(\mathfrak{K}_{j}\right)$, and — continuously — on the $y_{p}$ 's, and has positive leading coefficient. The assertion is now immediate.

Lemma 6.3. Let $\{1, \ldots, r+1\}=M_{1} \cup M_{2} \cup M_{3}$ be a partition of $\{1, \ldots$, $r+1\}$ into three (possibly empty) subsets. For $x \in \mathbb{R}_{+}^{r+1}$ and $\varepsilon>0$, let

$$
\begin{aligned}
S_{k}^{*}\left(x, \varepsilon ;\left(\mathfrak{K}_{j}\right), M_{1}, M_{2}, M_{3}\right)= & X \sum_{\hat{\nu}}^{\prime} d_{k}\left(\widehat{\nu} ;\left(\mathfrak{K}_{j}\right)\right) \\
& \times \prod_{p \in M_{1}}\left\{e^{-4 \varepsilon\left|\hat{\nu}^{(p)} x_{p}\right|^{2 / k}}\left|\widehat{\nu}^{(p)} x_{p}\right|^{-\left(e_{p} k+1\right) /(2 k)}\right\} \\
& \times \prod_{p \in M_{2}}\left\{e^{-2 \varepsilon\left|\hat{\nu}^{(p)} x_{p}\right|^{2 / k}}\left|\widehat{\nu}^{(p)} x_{p}\right|^{-\left(e_{p} k+2\right) /(2 k)}\right\} \\
& \times \prod_{p \in M_{3}}\left\{\left(\left|\widehat{\nu}^{(p)} x_{p}\right|+1\right)^{\left.-e_{p}\left|\widehat{\nu}^{(p)} x_{p}\right|^{-e_{p}}\right\} .}\right.
\end{aligned}
$$

Then, provided $M_{2} \cup M_{3} \neq \emptyset$,

$$
S_{k}^{*}\left(x, \varepsilon ;\left(\mathfrak{K}_{j}\right), M_{1}, M_{2}, M_{3}\right) \ll\left(\varepsilon^{n k / 2} X\right)^{-\delta} \varepsilon^{-(n k-r-2) / 4}
$$

for $0<\varepsilon \leq 1$ and any $\delta$ with $0<\delta<1$. The $\ll-$ constant depends only on $K, k$, and $\delta$.

Proof. The series can be reduced to the one dealt with in Lemma 6.1 by putting $T_{1}=M_{1} \cup M_{2}, T_{2}=M_{3}$, and

$$
y_{p}=\left(e_{p} k+l\right) /\left(2 e_{p} k\right) \quad \text { if } p \in M_{l}(l=1,2) .
$$

Let

$$
a=\frac{k}{2} \sum_{p \in T_{1}} e_{p} y_{p}, \quad b=\frac{k}{2} \sum_{p \in T_{1}} e_{p}
$$

then

$$
S_{k}^{*}\left(x, \varepsilon ;\left(\mathfrak{K}_{j}\right), M_{1}, M_{2}, M_{3}\right)=\prod_{p \in M_{1}} 4^{\left(e_{p} k+1\right) / 4} \cdot \prod_{p \in M_{2}} 2^{\left(e_{p} k+2\right) / 4} \cdot \varepsilon^{a} X S(\widetilde{x}),
$$

where

By Lemma 6.1,

$$
\widetilde{x}_{p}= \begin{cases}(4 \varepsilon)^{k / 2} x_{p} & \text { if } p \in M_{1} \\ (2 \varepsilon)^{k / 2} x_{p} & \text { if } p \in M_{2}, \\ x_{p} & \text { if } p \in M_{3}\end{cases}
$$

$$
S(\widetilde{x}) \ll \widetilde{X}^{-1-\delta} \ll\left(\varepsilon^{b} X\right)^{-1-\delta},
$$


hence

$$
S_{k}^{*}\left(x, \varepsilon ;\left(\mathfrak{K}_{j}\right), M_{1}, M_{2}, M_{3}\right) \ll\left(\varepsilon^{b} X\right)^{-\delta} \varepsilon^{-(b-a)},
$$

and the assertion follows since $b \leq n k / 2$ and

$$
4(b-a)=n k-r-1-\left|M_{2}\right|-\sum_{p \in M_{3}}\left(e_{p} k-1\right) .
$$

Lemma 6.4. For $x \in \mathbb{R}_{+}^{r+1}$ and $\varepsilon>0$, let

$$
S_{k}^{*}\left(x, \varepsilon ;\left(\mathfrak{K}_{j}\right)\right)=X \sum_{\hat{\nu}}^{\prime} d_{k}\left(\widehat{\nu} ;\left(\mathfrak{K}_{j}\right)\right) \prod_{p=1}^{r+1}\left\{e^{-4 \varepsilon\left|\hat{\nu}^{(p)} x_{p}\right|^{2 / k}}\left|\widehat{\nu}^{(p)} x_{p}\right|^{-\left(e_{p} k+1\right) /(2 k)}\right\} .
$$

Then

$$
S_{k}^{*}\left(x, \varepsilon ;\left(\mathfrak{K}_{j}\right)\right) \ll \varepsilon^{-(n k-r-1) / 4}\left(\left|\log \left(\varepsilon^{n k / 2} X\right)\right|+1\right)^{k-1},
$$

and, if $\varepsilon^{n k / 2} X \leq c_{2}$, also

$$
S_{k}^{*}\left(x, \varepsilon ;\left(\mathfrak{K}_{j}\right)\right) \gg \varepsilon^{-(n k-r-1) / 4}\left|\log \left(\varepsilon^{n k / 2} X\right)\right|^{k-1} .
$$

The constant $c_{2}, 0<c_{2} \leq 1 / 2$, and the constants implied by the symbols $\ll$ and $\gg$ depend only on $K$ and $k$.

Proof. Again, the assertion follows as a consequence of

$$
S_{k}^{*}\left(x, \varepsilon ;\left(\mathfrak{K}_{j}\right)\right)=\prod_{p=1}^{r+1}(4 \varepsilon)^{\left(e_{p} k+1\right) / 4} \cdot X S\left((4 \varepsilon)^{k / 2} x\right),
$$

where $S$ is the series considered in Lemma 6.2 with

$$
y_{p}=\left(e_{p} k+1\right) /\left(2 e_{p} k\right) \leq 3 / 4 \quad(p=1, \ldots, r+1) .
$$

7. A Voronoŭ-type equation. Once we have introduced ideal numbers, there is no advantage in restricting ourselves to the principal class; so we extend our earlier definition of $D_{k}\left(x ;\left(\mathfrak{K}_{j}\right)\right)$ to arbitrary systems $\left(\mathfrak{K}_{j}\right)=$ $\left(\mathfrak{K}_{1}, \ldots, \mathfrak{K}_{k}\right)$ of classes by setting

$$
D_{k}\left(x ;\left(\mathfrak{K}_{j}\right)\right)=\sum_{\left|\hat{\nu}^{(p)}\right| \leq x_{p}}^{\prime} d_{k}\left(\widehat{\nu} ;\left(\mathfrak{K}_{j}\right)\right),
$$

where the sum is over all ideal integers $\widehat{\nu} \neq 0$ in the region $\left|\widehat{\nu}^{(p)}\right| \leq x_{p}$ $(p=1, \ldots, r+1)$.

The main term in the asymptotic expansion of $D_{k}\left(x ;\left(\mathfrak{K}_{j}\right)\right)$ will turn out to be

$$
M_{k}\left(x ;\left(\mathfrak{K}_{j}\right)\right)=\operatorname{Res}_{s=1}\left(s^{-(r+1)} Z_{k}\left(s ; 1,\left(\mathfrak{K}_{j}\right)\right) X^{s}\right),
$$

which, by Lemma 4.1, is of the form

$$
M_{k}\left(x ;\left(\mathfrak{K}_{j}\right)\right)=\frac{1}{(k-1) !}\left(\frac{2^{r_{1}}(2 \pi)^{r_{2}}}{\sqrt{|d|}}\right)^{k}\left(\frac{R}{w}\right)^{k-1} X P_{k}\left(\log X ;\left(\mathfrak{K}_{j}\right)\right)
$$

with a monic polynomial $P_{k}\left(\cdot ;\left(\mathfrak{K}_{j}\right)\right)$ of degree $k-1$. 
We shall now establish an equation connecting the remainder

$$
\Delta_{k}\left(x ;\left(\mathfrak{K}_{j}\right)\right)=D_{k}\left(x ;\left(\mathfrak{K}_{j}\right)\right)-M_{k}\left(x ;\left(\mathfrak{K}_{j}\right)\right)
$$

with an infinite series, analogous to the Voronoŭ summation formula.

Lemma 7.1. For $x \in \mathbb{R}_{+}^{r+1}$ and $\varepsilon>0$, let

$$
\begin{aligned}
S_{k}\left(x, \varepsilon ;\left(\mathfrak{K}_{j}\right)\right)= & \left(\frac{-1}{\sqrt{k \pi}}\right)^{r+1}\left(\frac{4^{r_{2}} \pi^{n}}{|d|}\right)^{k / 2} X \sum_{\hat{\nu}}^{\prime} d_{k}\left(\widehat{\nu} ;\left(\mathfrak{K}_{j}^{\prime}\right)\right) \\
& \times \prod_{p=1}^{r+1}\left\{e^{-4 \varepsilon\left(C_{p}\left|\hat{\nu}^{(p)}\right| x_{p}\right)^{2 / k}}\left(C_{p}\left|\widehat{\nu}^{(p)}\right| x_{p}\right)^{-\left(e_{p} k+1\right) /(2 k)}\right. \\
& \left.\times \cos \left(2 k\left(C_{p}\left|\widehat{\nu}^{(p)}\right| x_{p}\right)^{1 / k}+\left(2-e_{p}\right) \frac{k \pi}{4}+\frac{\pi}{4}\right)\right\},
\end{aligned}
$$

where $\left(\mathfrak{K}_{j}^{\prime}\right)$ is the system of classes

$$
\mathfrak{K}_{j}^{\prime}=\mathfrak{K}_{j}^{-1} \cdot \mathfrak{K}(\widehat{\delta}) \quad(j=1, \ldots, k),
$$

and

$$
C_{p}=\left(e_{p} \pi /\left|\widehat{\delta}^{(p)}\right|\right)^{k} \quad(p=1, \ldots, r+1),
$$

$\widehat{\delta} \in \mathfrak{Z}$ being chosen such that $(\widehat{\delta})=\mathfrak{d}$ is the different of $K$. The series is absolutely convergent. For $x \in \mathbb{R}_{+}^{r+1}, 0<\varepsilon \leq 1 / 2$, and $0<\delta<1$, the following equality holds:

$$
\mathcal{J}_{\varepsilon} \Delta_{k}\left(x ;\left(\mathfrak{K}_{j}\right)\right)=S_{k}\left(x, \varepsilon ;\left(\mathfrak{K}_{j}\right)\right)+O\left(\left(\varepsilon^{n k / 2} X\right)^{-\delta} \varepsilon^{-(n k-r-2) / 4}\right)+O(1) .
$$

The $O$-constants depend only on $K, k$, and $\delta$.

Proof. We apply Lemma 3.1. As long as the general requirements of Section 3 are met, the particular choice of the group $\mathcal{U}$ is immaterial. For $\Phi$ we take the characteristic function of the unit cube $(0,1]^{r+1}$; then

$$
D_{k}\left(x ;\left(\mathfrak{K}_{j}\right)\right)=\sum_{\hat{\nu}}^{\prime} d_{k}\left(\widehat{\nu} ;\left(\mathfrak{K}_{j}\right)\right) \Phi\left(|\widehat{\nu}| x^{-1}\right),
$$

where $x^{-1}:=\left(x_{1}^{-1}, \ldots, x_{r+1}^{-1}\right)$. The Mellin transform of $\Phi$, for $\operatorname{Re} s_{p}>0$, is $\Psi\left(s_{1}, \ldots, s_{r+1}\right)=\left(s_{1} \ldots s_{r+1}\right)^{-1}$.

Thus, by Lemma 3.1 with $\tau=0$ and $x^{-1}$ in place of $x$, we have for $\sigma>1$,

$$
\begin{aligned}
\mathcal{J}_{\varepsilon} D_{k}\left(x ;\left(\mathfrak{K}_{j}\right)\right)= & \frac{1}{2 \pi i} \sum_{m} \int_{(\sigma)} \prod_{p=1}^{r+1} \frac{1}{s-i E_{p}(m)} \cdot Z_{k}\left(s ; \lambda_{m},\left(\mathfrak{K}_{j}\right)\right) \\
& \times \prod_{p=1}^{r+1} x_{p}^{e_{p}\left(s-i E_{p}(m)\right)} \cdot \exp \left\{\varepsilon \sum_{p=1}^{r+1} e_{p}^{2}\left(s-i E_{p}(m)\right)^{2}\right\} d s .
\end{aligned}
$$

If $m \neq 0$, the integrand is an entire function: the poles of $\prod_{p=1}^{r+1}\left(s-i E_{p}(m)\right)^{-1}$ are cancelled by trivial zeros of $Z_{k}$ which are exhibited 
in the functional equation (4.1). (The situation changes completely if the terms in the sum (7.1) are weighted by a sign character or even a genuine Grössencharacter: the trivial zeros are then no longer located in the right places, and an infinity of unpleasant residues occurs.)

Now let $m=0$. Then the integrand is holomorphic everywhere except at $s=1$ and possibly at $s=0$. The residue at $s=1 \mathrm{can}$, by Lemma 2.4, be put in the form $\mathcal{J}_{\varepsilon} M_{k}\left(x ;\left(\mathfrak{K}_{j}\right)\right)$ with $M_{k}$ given by (7.2). As is easily concluded from (4.1), $Z_{k}\left(s ; 1,\left(\mathfrak{K}_{j}\right)\right)$ has a zero of order $r k$ (i.e., no zero if $r=0$ ) at $s=0$. Hence the point $s=0$ is a simple pole for the integrand if $r=0$, and a regular point otherwise. In any case, the contribution of $s=0$ is $\ll 1$.

In view of Lemma 4.2, we may now move the path of integration to the left, across the critical strip, to the line $\operatorname{Re} s=-\sigma$, where $\sigma>0$. Since $\mathcal{J}_{\varepsilon}$ is a linear operator, it follows that

$$
\begin{aligned}
\mathcal{J}_{\varepsilon} \Delta_{k}\left(x ;\left(\mathfrak{K}_{j}\right)\right)+O(1)= & \frac{1}{2 \pi i} \sum_{m} \int_{(-\sigma)} \prod_{p=1}^{r+1} \frac{1}{s-i E_{p}(m)} \cdot Z_{k}\left(s ; \lambda_{m},\left(\mathfrak{K}_{j}\right)\right) \\
& \times \prod_{p=1}^{r+1} x_{p}^{e_{p}\left(s-i E_{p}(m)\right)} \cdot \exp \left\{\varepsilon \sum_{p=1}^{r+1} e_{p}^{2}\left(s-i E_{p}(m)\right)^{2}\right\} d s .
\end{aligned}
$$

Here we apply the functional equation (4.1) and make the change of variables $s \rightarrow-s, m \rightarrow-m$. Then the right-hand side becomes

$$
\begin{aligned}
\left(\frac{|d|}{4^{r_{2}} \pi^{n}}\right)^{k / 2} \frac{1}{2 \pi i} \sum_{m} \int_{(\sigma)} & \prod_{p=1}^{r+1}\left\{\frac{-1}{s-i E_{p}(m)}\left(\frac{\Gamma\left(\frac{e_{p}}{2}\left(1+s-i E_{p}(m)\right)\right)}{\Gamma\left(-\frac{e_{p}}{2}\left(s-i E_{p}(m)\right)\right)}\right)^{k}\right\} \\
& \times Z_{k}\left(1+s ; \lambda_{m},\left(\mathfrak{K}_{j}^{\prime}\right)\right) \prod_{p=1}^{r+1}\left(C_{p} x_{p}\right)^{-e_{p}\left(s-i E_{p}(m)\right)} \\
& \times \exp \left\{\varepsilon \sum_{p=1}^{r+1} e_{p}^{2}\left(s-i E_{p}(m)\right)^{2}\right\} d s .
\end{aligned}
$$

In the next step we employ the summation formula once more, this time in the version of Lemma 3.2, in order to get back a sum over numbers. In the notation of Lemma 3.2, we put

$$
f(\widehat{\nu})=d_{k}\left(\widehat{\nu} ;\left(\mathfrak{K}_{j}^{\prime}\right)\right) /|N(\widehat{\nu})| \quad \text { and } \quad \Psi\left(s_{1}, \ldots, s_{r+1}\right)=2^{r_{2}} \prod_{p=1}^{r+1} \psi_{p}\left(e_{p} s_{p}\right)
$$

with

$$
\psi_{p}(s)=\frac{1}{s}\left(\frac{\Gamma\left(\left(s+e_{p}\right) / 2\right)}{\Gamma(-s / 2)}\right)^{k} \quad(p=1, \ldots, r+1) .
$$


Then

$$
\widetilde{\Phi}_{\varepsilon}(u)=\prod_{p=1}^{r+1}\left\{\frac{e_{p}}{2 \pi i} \int_{(\sigma)} \psi_{p}\left(e_{p} s\right) u_{p}^{-e_{p} s} e^{\varepsilon e_{p}^{2} s^{2}} d s\right\}=\prod_{p=1}^{r+1} I_{\varepsilon}\left(u_{p} ; \psi_{p}\right),
$$

where $I_{\varepsilon}$ is the integral defined by (5.1). Hence, by Lemma 3.2,

$$
\begin{aligned}
& \mathcal{J}_{\varepsilon} \Delta_{k}\left(x ;\left(\mathfrak{K}_{j}\right)\right)+O(1) \\
&=(-1)^{r+1}\left(\frac{|d|}{4^{r_{2}} \pi^{n}}\right)^{k / 2} \sum_{\hat{\nu}}^{\prime} \frac{d_{k}\left(\widehat{\nu} ;\left(\mathfrak{K}_{j}^{\prime}\right)\right)}{|N(\widehat{\nu})|} \prod_{p=1}^{r+1} I_{\varepsilon}\left(C_{p}\left|\widehat{\nu}^{(p)}\right| x_{p} ; \psi_{p}\right),
\end{aligned}
$$

provided that the series is absolutely convergent, which we will prove now. By Lemma 5.3, applied with $a=\kappa=e_{p}$, we have for $B>0$ and $0<\varepsilon \leq 1 / 2$,

$$
\begin{aligned}
B^{-e_{p}} I_{\varepsilon}\left(B ; \psi_{p}\right)= & \frac{1}{\sqrt{k \pi}} e^{-4 \varepsilon B^{2 / k}} B^{-\left(e_{p} k+1\right) /(2 k)} \\
& \times \cos \left(2 k B^{1 / k}+\left(2-e_{p}\right) \frac{k \pi}{4}+\frac{\pi}{4}\right) \\
& +O\left(e^{-2 \varepsilon B^{2 / k}} B^{-\left(e_{p} k+2\right) /(2 k)}\right)+O\left((B+1)^{-e_{p}} B^{-e_{p}}\right) .
\end{aligned}
$$

Inserting this and multiplying, and observing that

$$
\prod_{p=1}^{r+1} C_{p}^{e_{p}}=\left(4^{r_{2}} \pi^{n} /|d|\right)^{k}
$$

we obtain

$\mathcal{J}_{\varepsilon} \Delta_{k}\left(x ;\left(\mathfrak{K}_{j}\right)\right)=S_{k}\left(x, \varepsilon ;\left(\mathfrak{K}_{j}\right)\right)+O(1)+O\left(\sum S_{k}^{*}\left(x^{\prime}, \varepsilon ;\left(\mathfrak{K}_{j}^{\prime}\right), M_{1}, M_{2}, M_{3}\right)\right)$,

where the sum $\sum$ is over all partitions of $\{1, \ldots, r+1\}$ into three subsets $M_{1}, M_{2}, M_{3}$ such that $M_{2} \cup M_{3} \neq \emptyset$. The series $S_{k}^{*}$ are those considered in Lemma 6.3 , taken at the point $x^{\prime}=\left(C_{1} x_{1}, \ldots, C_{r+1} x_{r+1}\right)$. Hence, the second $O$-term is

$$
\ll\left(\varepsilon^{n k / 2} X\right)^{-\delta} \varepsilon^{-(n k-r-2) / 4} .
$$

Finally, $S_{k}\left(x, \varepsilon ;\left(\mathfrak{K}_{j}\right)\right)$ is majorized by a constant multiple of $S_{k}^{*}\left(x^{\prime}, \varepsilon ;\left(\mathfrak{K}_{j}^{\prime}\right)\right)$ and thus, by Lemma 6.4, absolutely convergent.

It also follows that

$$
S_{k}\left(x, \varepsilon ;\left(\mathfrak{K}_{j}\right)\right) \ll \varepsilon^{-(n k-r-1) / 4}\left(\left|\log \left(\varepsilon^{n k / 2} X\right)\right|+1\right)^{k-1} ;
$$

we shall use this inequality in the next section.

Further, as an immediate consequence of (1.5), there is a corresponding Voronol formula for $\Delta_{k}(x)$, namely

$$
\mathcal{J}_{\varepsilon} \Delta_{k}(x)=S_{k}(x, \varepsilon)+O\left(\left(\varepsilon^{n k / 2} X\right)^{-\delta} \varepsilon^{-(n k-r-2) / 4}\right)+O(1),
$$


valid under the same conditions. Here $S_{k}(x, \varepsilon)$ is defined like $S_{k}\left(x, \varepsilon ;\left(\mathfrak{K}_{j}\right)\right)$, with $d_{k}\left(\widehat{\nu} ;\left(\mathfrak{K}_{j}^{\prime}\right)\right)$ replaced by $d_{k}(\widehat{\nu})=d_{k}((\widehat{\nu}))$ and the summation restricted to the integers $\widehat{\nu} \neq 0$ in the class $\mathfrak{K}\left(\widehat{\delta}^{k}\right)$.

8. Proof of Theorem 1. By Lemma 7.1 (with $\delta=(2 n k)^{-1}$ ) and (7.3), we have for $x \in \mathbb{R}_{+}^{r+1}$ and $0<\varepsilon \leq 1 / 2$,

$$
\mathcal{J}_{\varepsilon} \Delta_{k}\left(x ;\left(\mathfrak{K}_{j}\right)\right) \ll \varepsilon^{-(n k-r-1) / 4}\left\{(|\log X|+|\log \varepsilon|)^{k-1}+X^{-1 /(2 n k)}\right\} .
$$

We now choose

$$
\begin{aligned}
& \alpha(X)=(X+1)^{1-2 /(n k-r+1)}\left\{(|\log X|+1)^{k-1}+X^{-1 /(2 n k)}\right\}, \\
& \beta(X)=X(|\log X|+1)^{k-1}, \\
& \varepsilon(X)=\frac{1}{2}(X+1)^{-4 /(n k-r+1)} .
\end{aligned}
$$

Then $\alpha$ and $\beta$ are moderately growing functions, and

$$
\begin{aligned}
x_{p} \frac{\partial}{\partial x_{p}} M_{k}\left(x ;\left(\mathfrak{K}_{j}\right)\right) & =e_{p} A_{k} X\left\{P_{k}\left(\log X ;\left(\mathfrak{K}_{j}\right)\right)+P_{k}^{\prime}\left(\log X ;\left(\mathfrak{K}_{j}\right)\right)\right\} \\
& \ll \beta(X) \quad \text { for } p=1, \ldots, r+1 .
\end{aligned}
$$

Moreover, $\sqrt{\varepsilon(X)} \beta(X) \ll \alpha(X)$, and the right-hand side of (8.1), when taken at $\varepsilon=\varepsilon(X)$, is $\ll \alpha(X)$, too. Hence, by Lemma 2.2,

$$
\Delta_{k}\left(x ;\left(\mathfrak{K}_{j}\right)\right) \ll \alpha(X),
$$

and, by (1.5), the same holds for $\Delta_{k}(x)$. For $X \geq 2$, the assertion follows.

9. Proof of Theorem 2. In the remaining sections, $c_{3}, \ldots, c_{32}$ denote positive constants which, as well as all constants implied by the symbols $\ll$ and $\gg$, depend only on $K$ and $k$. With $\left(\mathfrak{K}_{j}^{\prime}\right)$ defined as in Lemma 7.1, let $\mathcal{M}$ denote the set of integers $\widehat{\nu} \neq 0$ in $\mathfrak{K}_{1}^{\prime} \ldots \mathfrak{K}_{k}^{\prime}$ such that $d_{k}\left(\widehat{\nu} ;\left(\mathfrak{K}_{j}^{\prime}\right)\right) \neq 0$. We fix a number $\widehat{\mu} \in \mathcal{M}$ such that $|N(\widehat{\mu})|$ is minimal and divide $\mathcal{M}$ into the subsets

$$
\mathcal{M}^{\prime}=\left\{\widehat{\nu} \in \mathcal{M}:\left|\widehat{\nu}^{(p)}\right|=\left|\widehat{\mu}^{(p)}\right| \text { for } p=1, \ldots, n\right\}
$$

and $\mathcal{M}^{\prime \prime}=\mathcal{M} \backslash \mathcal{M}^{\prime}$. Accordingly, we split $S_{k}\left(x, \varepsilon ;\left(\mathfrak{K}_{j}\right)\right)$ into the part $S^{\prime}$ corresponding to $\widehat{\nu} \in \mathcal{M}^{\prime}$ and the remainder $S^{\prime \prime}$ :

$$
S_{k}\left(x, \varepsilon ;\left(\mathfrak{K}_{j}\right)\right)=S^{\prime}+S^{\prime \prime} .
$$

We will choose $x$ and $\varepsilon$ in such a way as to make $S^{\prime}$ the dominating part. By the inequality between arithmetic and geometric means, for $\widehat{\nu} \in \mathcal{M}$ we have

$$
\sum_{p=1}^{r+1} e_{p}\left(\frac{\left|\widehat{\nu}^{(p)}\right|}{\left|\widehat{\mu}^{(p)}\right|}\right)^{2 / k}=\sum_{p=1}^{n}\left(\frac{\left|\widehat{\nu}^{(p)}\right|}{\left|\widehat{\mu}^{(p)}\right|}\right)^{2 / k} \geq n
$$


with equality only for $\widehat{\nu} \in \mathcal{M}^{\prime}$. Hence, say,

$$
\sum_{p=1}^{r+1} e_{p}\left(\frac{\left|\widehat{\nu}^{(p)}\right|}{\left|\widehat{\mu}^{(p)}\right|}\right)^{2 / k} \geq n\left(1+2 c_{3}\right)^{2} \quad \text { for } \widehat{\nu} \in \mathcal{M}^{\prime \prime}
$$

since the values of the left-hand side, for $\widehat{\nu} \in \mathcal{M}$, form a discrete subset of $\mathbb{R}_{+}$which itself depends only on $k, \mathcal{M}$, and the choice of $\widehat{\mu}$.

Now, for $t \geq 2$, consider $x=\left(x_{1}, \ldots, x_{r+1}\right)$ with

$$
x_{p}=\frac{e_{p}^{k / 2}}{C_{p}\left|\widehat{\mu}^{(p)}\right|}\left(t+\vartheta_{p}\right)^{k}, \quad \text { where } 0 \leq \vartheta_{p} \leq 2(p=1, \ldots, r+1),
$$

and

$$
\varepsilon=T / t^{2}, \quad \text { where } T \geq 2 ;
$$

the $\vartheta_{p}$ 's and $T$ will be chosen later. Then

$$
c_{4} t^{k} \leq x_{p} \leq c_{5} t^{k} \quad(p=1, \ldots, r+1)
$$

and, if $t \geq 2 / c_{3}$,

$$
4 \varepsilon \sum_{p=1}^{r+1}\left(C_{p}\left|\widehat{\nu}^{(p)}\right| x_{p}\right)^{2 / k} \leq 4 n T\left(1+c_{3}\right)^{2}=: c_{6} T \quad \text { for } \widehat{\nu} \in \mathcal{M}^{\prime},
$$

whereas for $\widehat{\nu} \in \mathcal{M}^{\prime \prime}(9.1)$ yields

$$
Y:=4 \varepsilon \sum_{p=1}^{r+1}\left(C_{p}\left|\widehat{\nu}^{(p)}\right| x_{p}\right)^{2 / k} \geq 4 n T\left(1+2 c_{3}\right)^{2}=: \frac{c_{6}+c_{7}}{1-4 c_{7}} T,
$$

say, so that

$$
Y \geq\left(c_{6}+c_{7}\right) T+4 c_{7} Y
$$

Hence

$$
\begin{aligned}
S^{\prime \prime} \ll & e^{-\left(c_{6}+c_{7}\right) T} X \sum_{\hat{\nu} \in \mathcal{M}} d_{k}\left(\widehat{\nu} ;\left(\mathfrak{K}_{j}^{\prime}\right)\right) \\
& \times \prod_{p=1}^{r+1}\left\{e^{-4 c_{7} \varepsilon\left(C_{p}\left|\hat{\nu}^{(p)}\right| x_{p}\right)^{2 / k}}\left(C_{p}\left|\widehat{\nu}^{(p)}\right| x_{p}\right)^{-\left(e_{p} k+1\right) /(2 k)}\right\} \\
& \ll e^{-\left(c_{6}+c_{7}\right) T} S_{k}^{*}\left(x^{\prime}, c_{7} \varepsilon ;\left(\mathfrak{K}_{j}^{\prime}\right)\right),
\end{aligned}
$$

where $x^{\prime}=\left(C_{1} x_{1}, \ldots, C_{r+1} x_{r+1}\right)$. Thus, by Lemma 6.4 and (9.2),

$$
S^{\prime \prime} \ll e^{-\left(c_{6}+c_{7}\right) T} X^{(n k-r-1) /(2 n k)} .
$$

Now let $\xi \in\{-1,+1\}$ be fixed. As $\vartheta_{1}, \ldots, \vartheta_{r+1}$ run independently through $[0,2]$, the values of each term

$$
2 k\left(C_{p}\left|\widehat{\mu}^{(p)}\right| x_{p}\right)^{1 / k}=2 k \sqrt{e_{p}}\left(t+\vartheta_{p}\right)
$$


cover an interval of length $>2 \pi$. Thus, given $t$, we can always find $\vartheta_{p}$ 's such that

$$
(-1)^{r+1} \prod_{p=1}^{r+1} \cos \left(2 k\left(C_{p}\left|\widehat{\mu}^{(p)}\right| x_{p}\right)^{1 / k}+\left(2-e_{p}\right) \frac{k \pi}{4}+\frac{\pi}{4}\right)=\xi,
$$

hence

$$
\begin{aligned}
\xi S^{\prime} & \gg X \prod_{p=1}^{r+1}\left\{e^{-4 \varepsilon\left(C_{p}\left|\hat{\mu}^{(p)}\right| x_{p}\right)^{2 / k}}\left(C_{p}\left|\widehat{\mu}^{(p)}\right| x_{p}\right)^{-\left(e_{p} k+1\right) /(2 k)}\right\} \\
& \gg e^{-c_{6} T} X^{(n k-r-1) /(2 n k)}
\end{aligned}
$$

by (9.2) and (9.3), hence

$$
\xi S_{k}\left(x, \varepsilon ;\left(\mathfrak{K}_{j}\right)\right) \geq e^{-c_{6} T}\left\{c_{8}-c_{9} e^{-c_{7} T}\right\} X^{(n k-r-1) /(2 n k)} .
$$

We now choose $T=c_{10}$ so large that $c_{8}-c_{9} e^{-c_{7} T}>0$. Then application of Lemma 7.1 with $\delta=1 / 2$ yields the following result: There exist $x \in \mathbb{R}_{+}$ and $\varepsilon>0$ such that $X$ is arbitrarily large,

$$
c_{11} X^{-2 /(n k)} \leq \varepsilon \leq c_{12} X^{-2 /(n k)},
$$

and

$$
\xi \mathcal{J}_{\varepsilon} \Delta_{k}\left(x ;\left(\mathfrak{K}_{j}\right)\right) \geq c_{13} X^{(n k-r-1) /(2 n k)} .
$$

From this, the assertion in the case of $\Delta_{k}\left(x ;\left(\mathfrak{K}_{j}\right)\right)$ follows by Lemma 2.3 . The proof for $\Delta_{k}(x)$, based on (7.4), is virtually the same.

10. Proof of Theorem 3. We use Diophantine approximation to find values of $x$ such that many terms of $S_{k}\left(x, \varepsilon ;\left(\mathfrak{K}_{j}\right)\right)$ have the same sign.

Throughout, it is assumed that either $k \not \equiv 1(\bmod 4)$ or $r_{1}=0$ or both. We introduce a new parameter $\lambda \geq 1$ and divide the set $\mathcal{M}$ defined in the previous section into

$$
\mathcal{M}_{\lambda}^{\prime}=\left\{\widehat{\nu} \in \mathcal{M}:\left(C_{p}\left|\widehat{\nu}^{(p)}\right|\right)^{2 / k} \leq \lambda \text { for } p=1, \ldots, r+1\right\}
$$

and $\mathcal{M}_{\lambda}^{\prime \prime}=\mathcal{M} \backslash \mathcal{M}_{\lambda}^{\prime}$. Moreover, we put

$$
V=r_{1}\left[\frac{k-2}{4}\right]+r_{2}
$$

LEMma 10.1. For every $\lambda \geq 1$, there is a real number $t>0$ such that

$$
c_{14} \lambda^{n k / 2} \leq \log t \leq 2 c_{14} \lambda^{n k / 2}
$$

and

$$
(-1)^{V+r+1} \prod_{p=1}^{r+1} \cos \left(2 k\left(C_{p}\left|\widehat{\nu}^{(p)}\right|\right)^{1 / k} t+\left(2-e_{p}\right) \frac{k \pi}{4}+\frac{\pi}{4}\right) \geq c_{15}
$$

for all $\widehat{\nu} \in \mathcal{M}_{\lambda}^{\prime}$. 
P r o o f. Owing to the lattice structure carried by the integers of $\mathfrak{K}_{1}^{\prime} \ldots \mathfrak{K}_{k}^{\prime}$, we have $\left|\mathcal{M}_{\lambda}^{\prime}\right| \leq c_{16} \lambda^{n k / 2}$. Hence, by Dirichlet's approximation theorem [5, Satz 462], there is a number $t$ in the interval

$$
100^{(r+1) c_{16} \lambda^{n k / 2}} \leq t \leq 100^{2(r+1) c_{16} \lambda^{n k / 2}}
$$

such that

$$
\left\|\frac{k}{\pi}\left(C_{p}\left|\widehat{\nu}^{(p)}\right|\right)^{1 / k} t\right\| \leq \frac{1}{100} \quad \text { for } \widehat{\nu} \in \mathcal{M}_{\lambda}^{\prime} \text { and } p=1, \ldots, r+1,
$$

$\|u\|$ denoting the distance of $u \in \mathbb{R}$ from the nearest rational integer. Hence

$$
\begin{aligned}
\cos \left(2 k\left(C_{p}\left|\widehat{\nu}^{(p)}\right|\right)^{1 / k} t\right. & \left.+\left(2-e_{p}\right) \frac{k \pi}{4}+\frac{\pi}{4}\right) \\
= & \cos \left(\left(2-e_{p}\right) \frac{k \pi}{4}+\frac{\pi}{4} \pm 2 \pi\left\|\frac{k}{\pi}\left(C_{p}\left|\widehat{\nu}^{(p)}\right|\right)^{1 / k} t\right\|\right)
\end{aligned}
$$

differs from

$$
\cos \left(\left(2-e_{p}\right) \frac{k \pi}{4}+\frac{\pi}{4}\right)
$$

by at most $2 \pi / 100$ in absolute value. Now

$$
(-1)^{[(l+2) / 4]} \cos \left(\frac{l+1}{4} \pi\right) \geq \frac{1}{2} \sqrt{2} \quad \text { for } l \in \mathbb{Z}, l \not \equiv 1(\bmod 4),
$$

and our above assumptions guarantee that

$$
\left(2-e_{p}\right) k \not \equiv 1(\bmod 4) \quad \text { for } p=1, \ldots, r+1 .
$$

Hence

$$
\begin{aligned}
(-1)^{\left[\left(\left(2-e_{p}\right) k+2\right) / 4\right]} & \cos \left(2 k\left(C_{p}\left|\widehat{\nu}^{(p)}\right|\right)^{1 / k} t+\left(2-e_{p}\right) \frac{k \pi}{4}+\frac{\pi}{4}\right) \\
\geq & \frac{1}{2} \sqrt{2}-\frac{2 \pi}{100}>\frac{1}{2} \quad \text { for } \widehat{\nu} \in \mathcal{M}_{\lambda}^{\prime}, p=1, \ldots, r+1,
\end{aligned}
$$

and the assertion follows since

$$
\sum_{p=1}^{r+1}\left[\frac{\left(2-e_{p}\right) k+2}{4}\right]=r_{1}\left[\frac{k+2}{4}\right] \equiv V+r+1(\bmod 2) .
$$

Lemma 10.2. There are $x \in \mathbb{R}_{+}^{r+1}$ (even with $x_{1}=x_{2}=\ldots=x_{r+1}$ ) and $\varepsilon>0$ such that $X$ is arbitrarily large,

$$
\varepsilon=c_{17}(X \log X)^{-2 /(n k)},
$$

and

$$
(-1)^{V} S_{k}\left(x, \varepsilon ;\left(\mathfrak{K}_{j}\right)\right) \geq c_{18} \alpha_{0}(X),
$$

where

$$
\alpha_{0}(X)=(X \log X)^{(n k-r-1) /(2 n k)}(\log \log X)^{k-1} .
$$


Proof. With $t$ determined according to Lemma 10.1, let

$$
\begin{aligned}
x & =\left(x_{1}, \ldots, x_{r+1}\right)=\left(t^{k}, \ldots, t^{k}\right), \\
x^{\prime} & =\left(x_{1}^{\prime}, \ldots, x_{r+1}^{\prime}\right)=\left(C_{1} t^{k}, \ldots, C_{r+1} t^{k}\right),
\end{aligned}
$$

so that $X=t^{n k}, X^{\prime}=c_{19} X$, and $X, X^{\prime} \rightarrow \infty$ as $\lambda \rightarrow \infty$. Then

$$
\begin{aligned}
&(-1)^{V} S_{k}\left(x, \varepsilon ;\left(\mathfrak{K}_{j}\right)\right) \\
& \geq c_{20} X\left(c_{15} \sum_{\hat{\nu} \in \mathcal{M}_{\lambda}^{\prime}}-\sum_{\hat{\nu} \in \mathcal{M}_{\lambda}^{\prime \prime}}\right) d_{k}\left(\widehat{\nu} ;\left(\mathfrak{K}_{j}^{\prime}\right)\right) \\
& \times \prod_{p=1}^{r+1}\left\{e^{\left.-4 \varepsilon\left|\hat{\nu}^{(p)} x_{p}^{\prime}\right|^{2 / k}\left|\widehat{\nu}^{(p)} x_{p}^{\prime}\right|^{-\left(e_{p} k+1\right) /(2 k)}\right\}}\right. \\
&= c_{21} X^{\prime}\left(c_{15} \sum_{\hat{\nu} \in \mathcal{M}}-\left(1+c_{15}\right) \sum_{\hat{\nu} \in \mathcal{M}_{\lambda}^{\prime \prime}}\right) d_{k}\left(\widehat{\nu} ;\left(\mathfrak{K}_{j}^{\prime}\right)\right) \\
& \times \prod_{p=1}^{r+1}\left\{e^{\left.-4 \varepsilon\left|\hat{\nu}^{(p)} x_{p}^{\prime}\right|^{2 / k}\left|\widehat{\nu}^{(p)} x_{p}^{\prime}\right|^{-\left(e_{p} k+1\right) /(2 k)}\right\}}\right. \\
& \geq c_{21}\left(c_{15} S_{k}^{*}\left(x^{\prime}, \varepsilon ;\left(\mathfrak{K}_{j}^{\prime}\right)\right)-\left(1+c_{15}\right) e^{-\varepsilon \lambda t^{2}} S_{k}^{*}\left(x^{\prime}, \varepsilon / 2 ;\left(\mathfrak{K}_{j}^{\prime}\right)\right)\right)
\end{aligned}
$$

since

$$
2 \varepsilon \sum_{p=1}^{r+1}\left|\widehat{\nu}^{(p)} x_{p}^{\prime}\right|^{2 / k}>\varepsilon \lambda t^{2} \quad \text { for } \widehat{\nu} \in \mathcal{M}_{\lambda}^{\prime \prime} .
$$

Hence, by Lemma 6.4,

$$
(-1)^{V} S_{k}\left(x, \varepsilon ;\left(\mathfrak{K}_{j}\right)\right) \geq\left\{c_{22}-c_{23} e^{-\varepsilon \lambda t^{2}}\right\} \varepsilon^{-(n k-r-1) / 4}\left|\log \left(\varepsilon^{n k / 2} X\right)\right|^{k-1}
$$

provided $\varepsilon^{n k / 2} X \leq c_{24} \leq 1 / 2$, hence

$$
(-1)^{V} S_{k}\left(x, \varepsilon ;\left(\mathfrak{K}_{j}\right)\right) \geq c_{25} \varepsilon^{-(n k-r-1) / 4}\left|\log \left(\varepsilon^{n k / 2} X\right)\right|^{k-1}
$$

provided that also $\varepsilon \lambda t^{2} \geq c_{26}$, where $c_{22}-c_{23} e^{-c_{26}}>0$.

By (10.1),

$$
c_{27} \lambda \leq(\log X)^{2 /(n k)} \leq c_{28} \lambda .
$$

Now let $\varepsilon$ be given by (10.2) with the constant $c_{17}$ chosen as $c_{17}:=c_{26} c_{28}$. Then, if $\lambda$ is sufficiently large,

$$
\varepsilon^{n k / 2} X=c_{17}^{n k / 2}(\log X)^{-1} \leq c_{24}
$$

and, by (10.4),

$$
\varepsilon \lambda t^{2}=c_{17}(\log X)^{-2 /(n k)} \lambda \geq c_{17} / c_{28}=c_{26} .
$$


Thus, all conditions for the validity of (10.3) are met, and substituting $\varepsilon$ from (10.2) yields

$$
\begin{aligned}
& (-1)^{V} S_{k}\left(x, \varepsilon ;\left(\mathfrak{K}_{j}\right)\right) \\
& \quad \geq c_{29}(X \log X)^{(n k-r-1) /(2 n k)}\left|\log \left(c_{30} \log X\right)\right|^{k-1} \geq c_{18} \alpha_{0}(X)
\end{aligned}
$$

if $X$ is large enough.

To complete the proof of Theorem 3 in the case of $\Delta_{k}\left(x ;\left(\mathfrak{K}_{j}\right)\right)$, we apply Lemma 7.1 with $\delta=(2 n k)^{-1}$. If $x$ and $\varepsilon$ are chosen according to Lemma 10.2, and if $X$ is sufficiently large, we obtain

$$
(-1)^{V} \mathcal{J}_{\varepsilon} \Delta_{k}\left(x ;\left(\mathfrak{K}_{j}\right)\right) \geq c_{31} \alpha_{0}(X) .
$$

Here the right-hand side may as well be replaced by $c_{32} \alpha(X)$, where $\alpha(X):=$ $\alpha_{0}(X+10)$, both functions having the same order of magnitude as $X \rightarrow \infty$. However, $\alpha(X)$ has the advantage of being defined on the whole of $\mathbb{R}_{+}$, and of being moderately growing. Hence Lemma 2.3 is applicable, yielding the assertion.

The proof for $\Delta_{k}(x)$ proceeds in the same manner, starting from (7.4).

\section{References}

[1] W. Grotz, Einige Anwendungen der Siegelschen Summenformel, Acta Arith. 38 (1980), 69-95.

[2] G. H. Hardy, On Dirichlet's divisor problem, Proc. London Math. Soc. (2) 15 (1916), 1-25.

[3] E. Hecke, Eine neue Art von Zetafunktionen und ihre Beziehungen zur Verteilung der Primzahlen (Zweite Mitteilung), Math. Z. 6 (1920), 11-51.

[4] E. Landau, Über Dirichlets Teilerproblem, Sitzungsber. Math.-Phys. Kl. Königl. Bayer. Akad. Wiss. 1915, 317-328.

[5] -, Vorlesungen über Zahlentheorie, reprint, Chelsea, New York, 1969.

[6] - Einführung in die elementare und analytische Theorie der algebraischen Zahlen und der Ideale, reprint, Chelsea, New York, 1949.

[7] W. Narkiewicz, Elementary and Analytic Theory of Algebraic Numbers, 2nd ed., Springer, Berlin, 1990.

[8] H. Rademacher, On the Phragmén-Lindelöf theorem and some applications, Math. Z. 72 (1959), 192-204.

[9] U. Rausch, Eine Formel für die Koeffizientensumme von Potenzreihen mit Anwendung auf das Kreis- und Kugelproblem in total reellen algebraischen Zahlkörpern, Acta Arith. 50 (1988), 381-404.

[10] - - A summation formula in algebraic number fields and applications, I, J. Number Theory 36 (1990), 46-79.

[11] —, Zum Ellipsoidproblem in algebraischen Zahlkörpern, Acta Arith. 58 (1991), 309333.

[12] P. Söhne, Eine Abschätzung für Heckesche Zetafunktionen mit Größencharakteren auf der kritischen Geraden, Dissertation, Marburg, 1990. 
[13] P. Söhne, Some asymptotic formulae in algebraic number fields, to appear.

[14] G. Szegö und A. Walfisz, Über das Piltzsche Teilerproblem in algebraischen Zahlkörpern (Erste Abhandlung), Math. Z. 26 (1927), 138-156.

ABTEILUNG MATHEMATIK III

UNIVERSITÄT ULM

HELMHOLTZSTRASSE 18

D-89081 ULM, GERMANY

Received on 9.12.1993

and in revised form on 8.3.1994 